\title{
Geology and Geochemistry of Selected Gold Deposits in the Ailaoshan Metallogenic Belt, China: Origin of Ore-Forming Fluids
}

\author{
Yang Li ${ }^{1} \mathbb{D}$, Denghong Wang ${ }^{2}$, Chenghui Wang ${ }^{2, *}$, Yan Sun $^{2}$ and MIMA Pu-chi ${ }^{3}$ \\ 1 College of Earth Sciences, Chengdu University of Technology, Chengdu 610059, China; liyangcdut@163.com \\ 2 Institute of Mineral Resources, Chinese Academy of Geological Sciences, Beijing 100037, China; \\ wangdenghong@vip.sina.com (D.W.); sunyan517@vip.163.com (Y.S.) \\ 3 Bureau of Geology and Mineral Exploration and Development of Tibet Autonomous Region, \\ Regional Geological Survey Brigade, Lhasa 851400, China; mier_0102@163.com \\ * Correspondence: wangchenghui131@sina.com
}

check for

updates

Citation: Li, Y.; Wang, D.; Wang, C.; Sun, Y.; Pu-chi, M. Geology and Geochemistry of Selected Gold Deposits in the Ailaoshan Metallogenic Belt, China: Origin of Ore-Forming Fluids. Minerals 2021, 11, 1276. https://doi.org/10.3390/ $\min 11111276$

Academic Editors: Anastassia Yurievna Borisova and Stefano Salvi

Received: 2 September 2021

Accepted: 12 November 2021

Published: 18 November 2021

Publisher's Note: MDPI stays neutral with regard to jurisdictional claims in published maps and institutional affiliations.

Copyright: (c) 2021 by the authors. Licensee MDPI, Basel, Switzerland. This article is an open access article distributed under the terms and conditions of the Creative Commons Attribution (CC BY) license (https:// creativecommons.org/licenses/by/ $4.0 /)$.

\begin{abstract}
The formation of the Ailaoshan metallogenic belt was the result of: the Neoproterozoic super mantle plume, the Indosinian and South China blocks in the Late Triassic after the PaleoTethys Ocean closure, and Oligocene-Eocene continental-scale shearing related to the India-Eurasia collision. It is one of the most important Cenozoic gold ore province in the world. In this paper, the geological characteristics, isotopic geochemistry, and geochemical data of ore-forming fluids of four large-scale gold deposits in the Ailaoshan metallogenic belt (Mojiang Jinchang, Zhenyuan Laowangzhai, Yuanyang Daping, and Jinping Chang'an) are comprehensively compared. The features of host-rock alteration, metallogenetic periods and stages, geochronology, fluid inclusion, and $\mathrm{C}-\mathrm{H}-\mathrm{O}-\mathrm{S}-\mathrm{Pb}$ isotopes of gold deposits are summarized and analyzed. The gold mineralization in the Ailaoshan metallogenic belt occurred mostly in 50-30 Ma, belonging to the Himalayan period. The gold mineralization is closely related to silicification, argillation, carbonation, and pyritization due to the strong mineralization of hydrothermal fluid, the development of alteration products, and the inconspicuous spatial zonation of alteration types. The ore-forming fluid is mainly composed of mantle fluid (magmatic water) and metamorphic fluid (metamorphic water). The ore-forming materials of the Jinchang, Chang'an, and Laowangzhai gold deposits mainly originate the host-rock strata of the mining area, and the carbon is more likely to from marine carbonate. The carbon in the Daping gold deposit from the original magma formed by the partial melting of the mantle. $\mathrm{Pb}$ isotopes have characteristics of crustal origin, accompanied by mixing of mantle-derived materials and multisource sulfur mixing, and are strongly homogenized.
\end{abstract}

Keywords: the Ailaoshan metallogenic belt; geological characteristics of deposit; chronological characteristics; C-H-O-S-Pb isotopes

\section{Introduction}

The Ailaoshan metallogenic belt is a boundary tectonic-magmatic-metallogenic belt between the western margin of the Yangtze and Indosinian blocks. It encompasses the Ailaoshan-Red River shear zone (ASRRSZ) in the north east and the Ailaoshan suture zone in the north west (Wang et al., 2013; Liu et al., 2011) [1,2]. The Ailaoshan metallogenic belt is located in the southern section of the ASRRSZ (also known as the Ailaoshan fault zone), where the Yangtze plate meets the Indian plate. The Ailaoshan metallogenic belt has an overall broom-like geometric structure that converges to the northwest and expands to the southeast (Figure 1a, Metcalfe, 2013) [3]. It is divided into the eastern high-grade metamorphic belt typified by ductile shearing and the western low-grade metamorphic belt, which is further subdivided into the Jinping terrane in the South China block and a Paleo-Tethyan ophiolite belt in the Indochina block (Figure 1b). The Ailaoshan fault 
separates the eastern high-grade metamorphic belt from the Jinping terrane. The AilaoshanTengtiaohe fault separates the western low-grade metamorphic belt from the ophiolitic belt (Wang et al., 2020) [4,5].

The Ailaoshan metallogenic belt evolved during the last continental collision. Prior to this, multiple plate gatherings considerably affected this metallogenic belt (Schärer et al., 1990; Leloup et al., 1995; Hou and Cook., 2009; Hou et al., 2015) [6-9]. The formation of the Ailaoshan metallogenic belt was the result of: super mantle plume activity in the Neoproterozoic ( $\mathrm{Li}$ et al., 1999; Li et al., 2008; Li Xianhua et al., 2002; Zou et al., 2020) [10-13], the closure of the Paleo-Tethys Ocean in the Late Paleozoic (Liu et al., 2014, 2015; Yang et al., 2018) [14-16], Cenozoic India-Eurasia continental collision (Deng et al., 2014, 2017, Hou et al., 2015; Hou and Cook 2009) [8,9,17-19], and other multiple orogeny periods. Subsequently, a series of gold deposits formed in the complex crust above the abnormal mantle lithosphere (Figure 1b, Wang et al., 2020) [5]. From the NW to the SE, there are Mojiang Jinchang, Zhenyuan Laowangzhai, Zhenyuan Donggualin, Yuanyang Daping, Yuanyang Malutang, Jinping Tongchang, Jinping Chang'an, Xiangyun Jinchangqing, Heqing Beiya, Nanhua Yao'an, and other large- and medium-sized gold deposits as well as numerous small deposits (ore spots) (Li, 2014) [20]. Among the four major large-scale deposits (Zhenyuan Laowangzhai, Mojiang Jinchang, Jinping Chang'an, and Yuanyang Daping) (Sun et al., 2009) [21], except for the Yuanyang Daping gold deposit in a Hercynian diorite rock body (Zhao, 2014; Yang et al., 2011) [22,23], the rest are located in the Ailaoshan shallow metamorphic rock series (Deng et al., 2010a, b) [24,25]. The orebody of the gold deposit was controlled by the secondary fault structure of the regional NW-trending fault and formed in the process of Tethys composite orogeny.

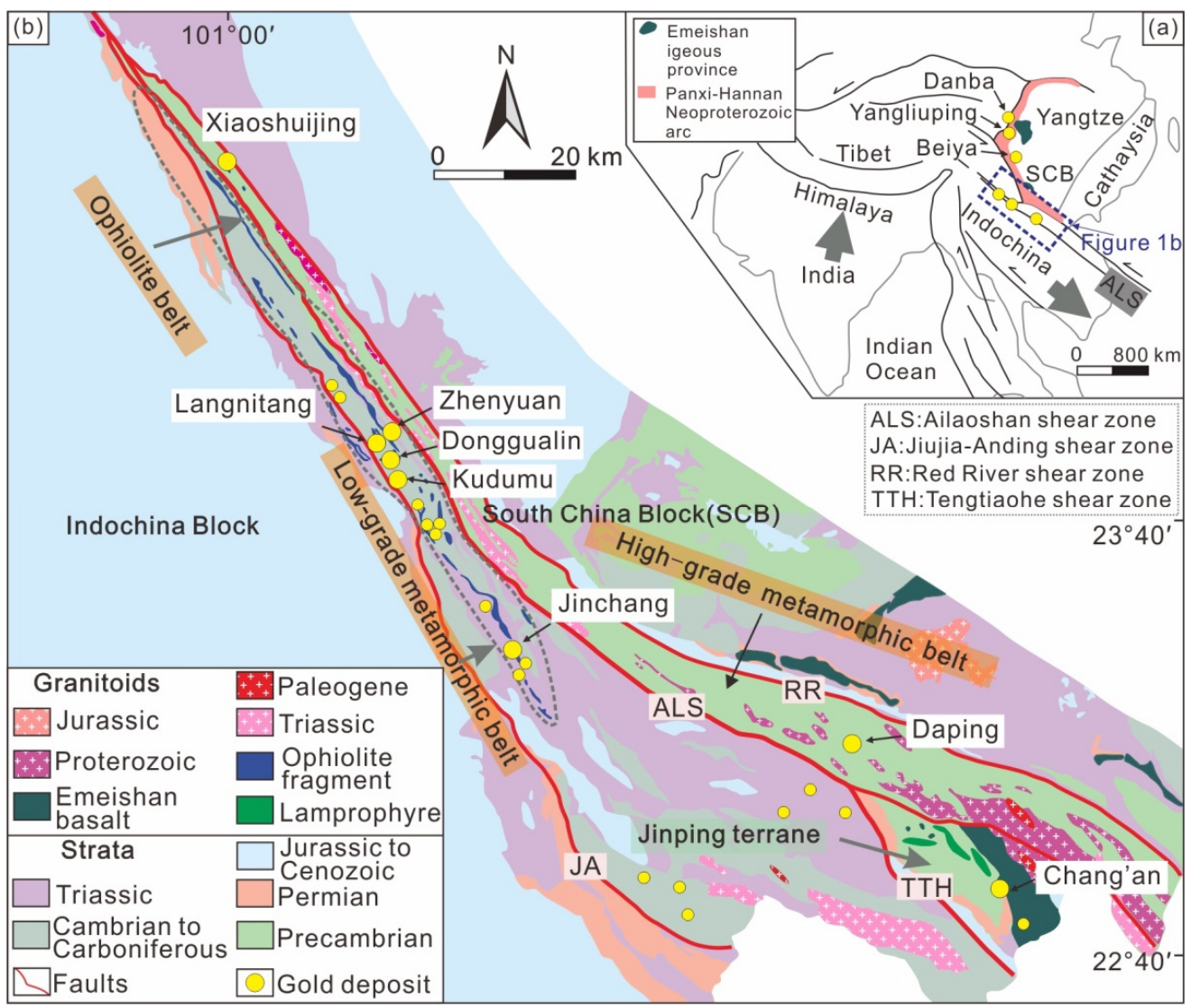

Figure 1. (a) Generalized geological map showing major tectonic structures caused by to the IndiaEurasia collision (revised from [4,26]) and (b) geological map of the Ailaoshan gold belt along the Ailaoshan shear zone showing the high-grade metamorphic belt, ophiolite belt, and Jinping terrane (modified from [17]). 
Many studies have been conducted in the area, obtaining important results (Goldfarb et al., 2001; Faure et al., 2016) $[27,28]$. Based on previous studies, this study selected four major large-scale gold deposits (Sun et al., 2009) [21] in the Ailaoshan metallogenic belt for conducting systematic research. The data were collected from previous works and reinterpreted. We applied C-H-O-S-Pb isotopic systems for determining diagenesis, the mineralization stages, and the study of the source and evolution of ore-forming fluids in the Ailaoshan ore belt. We focused on the metallogenic characteristics of the metallogenic belt, explored the geochemical characteristics of gold mineralization under the background of the Ailaoshan compound orogen, explored the genetic mechanism and metallogenic laws of the deposit, established the corresponding metallogenic model, and provided a reliable theoretical basis for guiding the investigation.

\section{Data Sources}

This paper is based on a review of previous studies. The temperature measurement data of fluid inclusions are mostly derived from quartz, with a small amount of calcite. According to the microscopic temperature measurement data, for $\mathrm{CO}_{2}-\mathrm{H}_{2} \mathrm{O}$ three-phase inclusions, use the formula for calculating the salinity of this type of inclusion:

$$
S=15.52022-1.02342 t-0.05286 t^{2}\left(-9.6^{\circ} \mathrm{C} \leq t \leq 10{ }^{\circ} \mathrm{C}\right) .
$$

In the formula, $S$ is the salinity ( $\mathrm{wt} \% \mathrm{NaCl}$. eqv.) and $t$ is the dissolution temperature of $\mathrm{CO}_{2}$ hydrate $\left({ }^{\circ} \mathrm{C}\right.$ ) (Bozzo et al., 1973) [29].

For aqueous inclusions, use the salinity calculation formula:

$$
S=0.00+1.78 t-0.0442 t^{2}+0.000557 t^{3}(0-23.3 \% \mathrm{NaCl} \text { solutions }) .
$$

In the formula, $S$ is the salinity ( $\mathrm{wt} \% \mathrm{NaCl}$. eqv.) and $t$ is the temperature at which the freezing point drops $\left({ }^{\circ} \mathrm{C}\right)$ (Lu et al., 2004) [30].

$\mathrm{S}$ isotope data mostly come from pyrite and stibnite, with a small amount of chalcopyrite, galena, barite, sulfur copper antimony, iron dolomite, and arsenopyrite. C-H-O isotope data mostly come from quartz and calcite, with a small amount of scheelite, dolomite, iron dolomite, and siderite. $\mathrm{Pb}$ isotope data mostly come from galena. The above isotope data are from single minerals in ore and host rocks. Section 4 (Metallogenic geodynamic background) show the dating minerals and methods.

\section{Geological Characteristics of Ailaoshan Gold Deposits}

\subsection{The Jinchang Deposit in Mojiang}

The Jinchang deposit, with a resource of more than $80 \mathrm{t}$ at $2.69 \mathrm{~g} / \mathrm{t}$ gold, has a unique gold-nickel paragenetic assemblage (Fang et al., 2001) [31]. The host rocks mainly comprise the Early-Middle Devonian metasandstone interbedded with slate, defining an overturned fold and widely exposed Late Triassic red siltstone, siliceous metasedimentary rocks, and a large amount of brecciated Devonian ophiolites (Figure 2a,b). The ophiolites represent partially serpentinized augite peridotite and dunite (Xiong et al., 2015) [32]. Northweststriking granitoid intrudes the Devonian-Triassic metasedimentary rocks and ophiolites (Figure 2a). In the geotectonic position, the Jinchang deposit is located near the contact zone between the Sanjiang orogenic belt and the Yangtze block and it is located in the contact zone between the Upper Devonian shallow metamorphic rock series on the east side of the Jiujia-Mojiang fault and the Jinchang ultrabasic rock pluton in the Ailaoshan ophiolitic mélange belt (Xiong et al., 2015) [32]. The mine is divided into five ore blocks in the following order: the Sishibaliangshan, Laojiniu, Lanshan, Pingpo, and Maobiliangz ore blocks (Figure 2; Xiong et al., 2015) [32]. 


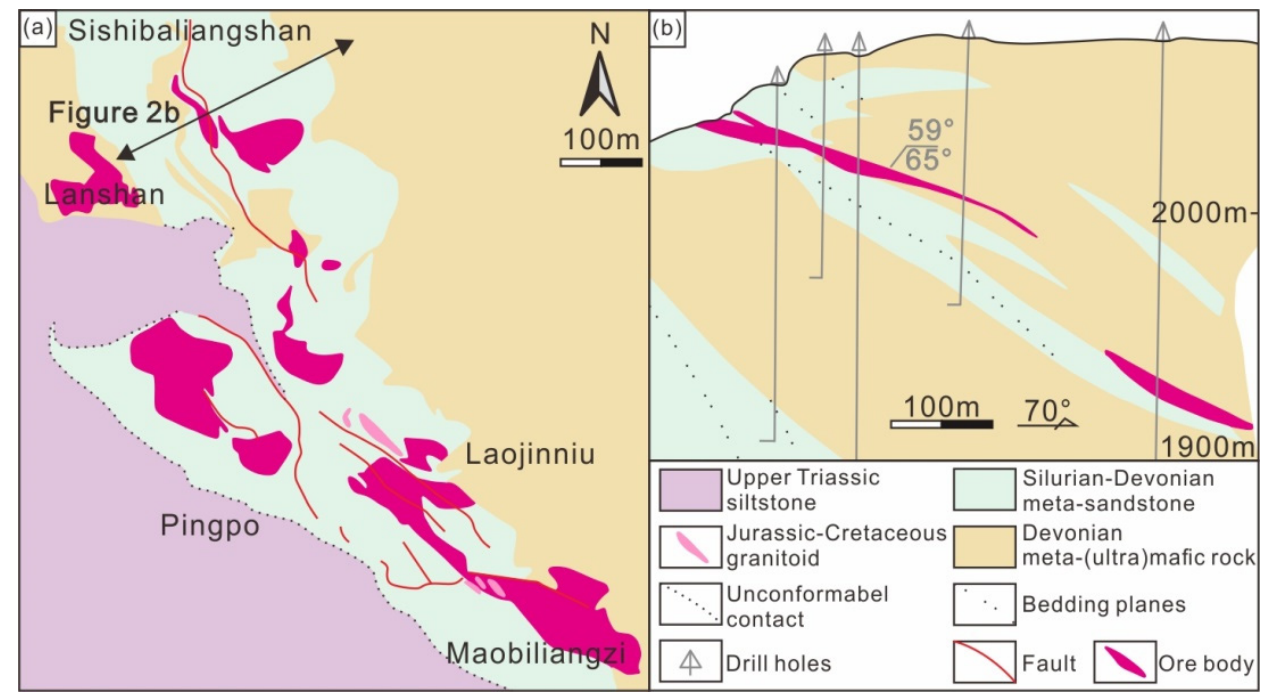

Figure 2. (a) Geological map of the Jinchang gold deposit showing granitoid, meta-(ultra) mafic rocks and ore bodies. (b) geological profile of Jinchang gold deposit exploration line (modified from [33]).

\subsection{The Zhenyuan Deposit}

From NW to SE, the Zhenyuan deposit, comprising the Langnitang, Laowangzhai, Donggualin, Daqiaoqing, and Kudumu ore-shoot clusters (Figure 3a,b, BGMRYP (Bureau of Geology and Mineral Resources of Yunnan Province), (1993a, b) [34,35], is the largest gold deposit in the belt, with a resource of more than $160 \mathrm{t}$ at $5.29 \mathrm{~g} / \mathrm{t}$ gold (Yang et al., 2010) [36]. The deposit is in the northern part of the ophiolite belt, characterized by complex host rocks, including Late Devonian metaquartz sandstone, Devonian-Carboniferous ophiolitic rocks, Carboniferous carbonaceous slate, Permo-Triassic quartz porphyry, Late Triassic siltstone, and Eocene lamprophyre (Deng et al., 2015a; Huang et al., 2002) [37,38]. Structural controls are dominated by combinations of faults, folds, and shear joints, with steep NW-striking faults in the Donggualin cluster and nearly EW-NE-striking faults in the Laowangzhai ore cluster (Deng et al., 2015a) [37].

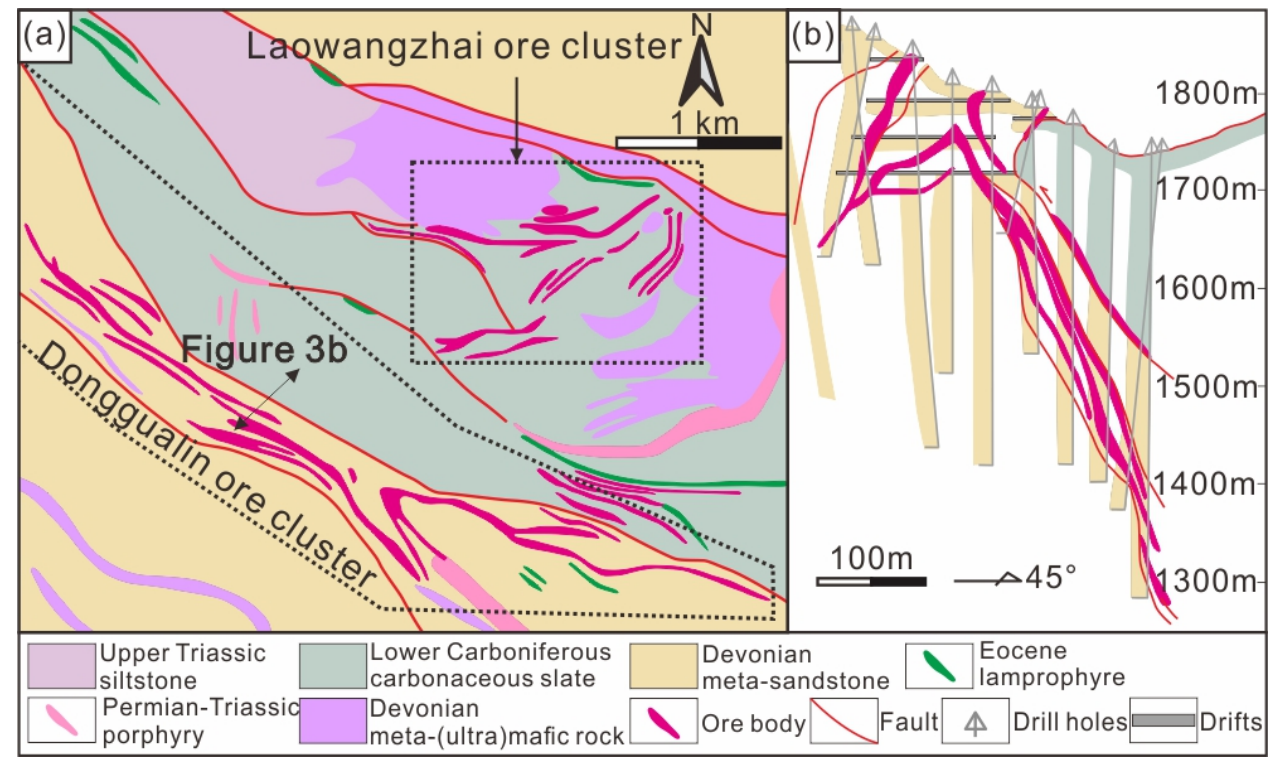

Figure 3. (a) Geological map and cross-section of the Zhenyuan gold deposit showing felsic, mafic, and lamprophyre dykes and gold ore bodies. (b) Drill sections from the Donggualin ore-shoot cluster of the Zhenyuan ore deposit (modified from $[4,34,35]$ ). 


\subsection{The Daping Deposit in Yuanyang}

The Yuanyang Daping gold deposit, with a resource of more than $60 \mathrm{t}$ at $14.3 \mathrm{~g} / \mathrm{t}$ gold (Yang et al., 2010) [36]. So far, 40 tons of gold have been mined. Many types of rocks are exposed in the area, among which the main host rocks are Neoproterozoic granite diorite and Silurian dolomite. Considering the Xiaoxinjie fault as the boundary, the Baishapo ore block is on the west side, the Maomushu ore block is on the north side, the Mashami ore block is in the middle section, and the Bajiaoling ore block is on the south side (Figure 4). Based on the spatial distribution of the veins and the relationship with the Xiaoxinjie fault, it can be inferred that the Xiaoxinjie fault is the main ore-conducting structure in the mining area (Yang, 2018) [39].

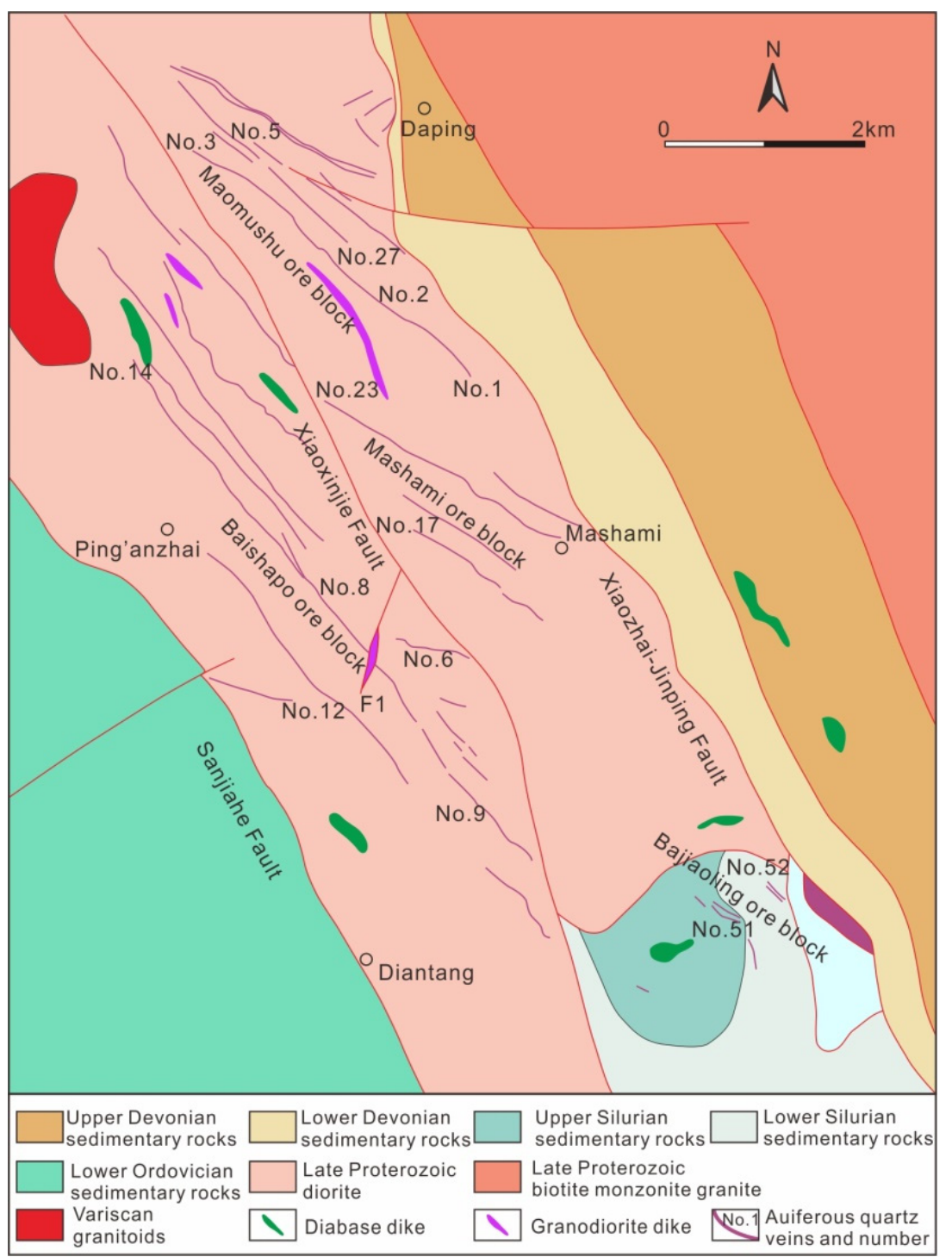

Figure 4. Geological map of Yuanyang Daping gold deposit (modified from [40]).

\subsection{The Chang'an Deposit in Jinping}

The Chang'an gold deposit, with more than $110 \mathrm{t}$ at $5.84 \mathrm{~g} / \mathrm{t}$ gold, is in the western part of the Jinping terrane (Figure 5; Yang et al., 2010) [36]. Rocks in this deposit mostly include Early Ordovician metamorphosed fine-grained quartz sandstone and quartz conglomerate, Middle-Late Silurian dolomite, and Quaternary gravel (Zhang et al., 2014) [41]. The Ordovician sandstone and conglomerate, the main host rocks, are approximately E-Wtrending and N-dipping (Zhang et al., 2014; Wang et al., 2020) [4,41]. 


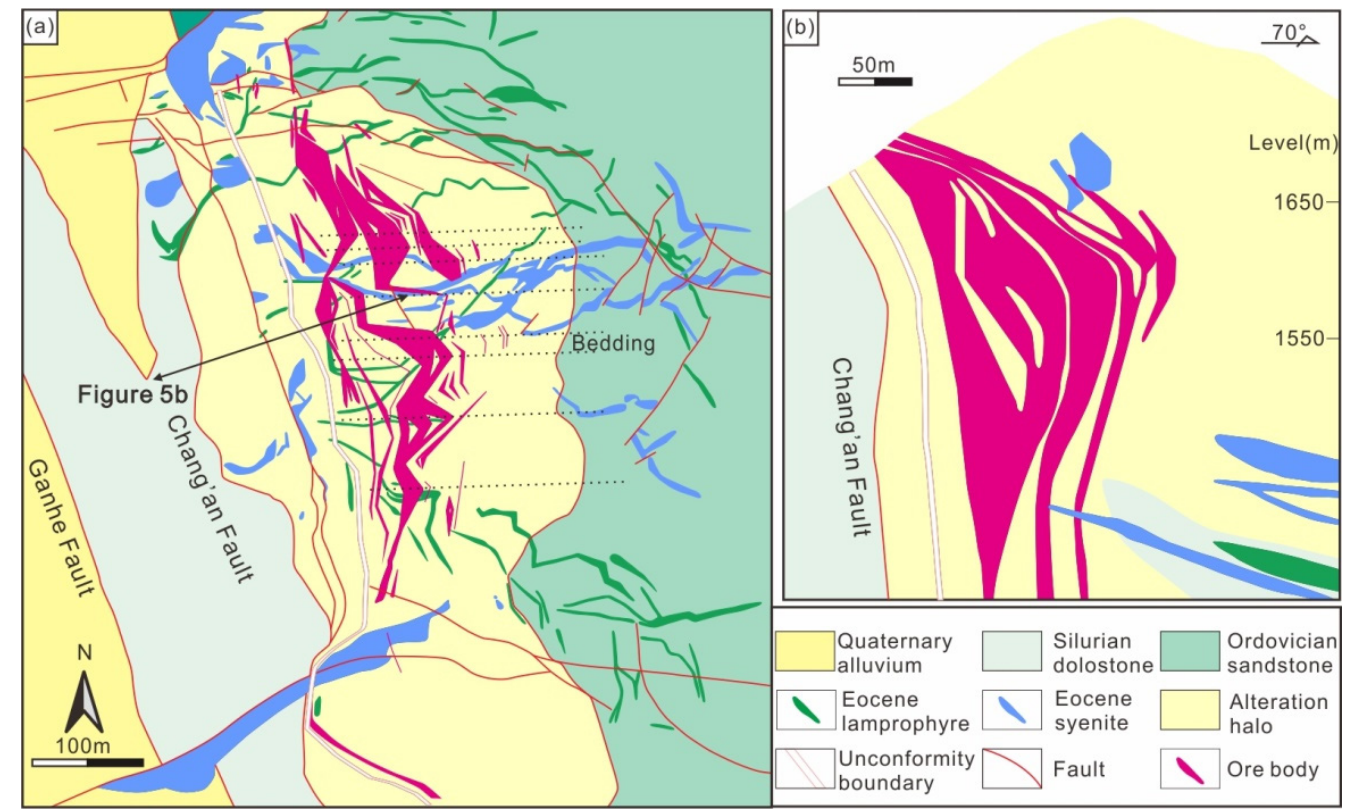

Figure 5. (a) Geological map showing lamprophyre and syenite dykes, widespread host-rock alteration, and gold ore bodies in the Chang'an gold deposit. (b) geological profile of Chang'an gold deposit exploration line (modified from [41]).

\subsection{Host-Rock Alteration and Mineralization Stages}

\subsubsection{Characteristics of Host-Rock Alteration}

The tectonic-magmatic activity in the Ailaoshan metallogenic belt is intense, forming a series of ultrabasic, basic, and intermediate-acid magmatic rocks. Most of the rock pluton is produced in the form of small rock plutons and dykes and distributed intermittently along the deep fault zone of the Ailaoshan. The Ailaoshan deep fault structural zone is mainly composed of magmatic rocks, and the fillings in the secondary fault zones are cataclastic rocks, mylonites, and hydrothermal veins. The hydrothermal veins are mostly quartz and calcite veins. The hydrothermal process is strong, and the alteration products are developed, mainly including silicification, sericitization (chromium hydromicaization), argillation, pyrite sericitization, pyritization, carbonation, iron dolomitization, and green mud petrification and serpentinization. The spatial zoning of alteration types is not obvious, and gold mineralization is closely related to silicification, clayization, carbonatization, and pyritization (Table 1, Zhao, 2014) [22].

\subsubsection{Mineralization Periods and Stages Division}

The mineralization of the four main gold deposits in the Ailaoshan metallogenic belt exhibits both common features and obvious differences. These features and differences relate to the complex geological processes developed in different periods of the metallogenic belt and the different metallogenic environments among the deposits. It is also controversial whether the four deposits exhibit multistage mineralization.

Based on previous viewpoints and hand specimen and microscopic identification, the main metallogenic periods in the metallogenic belt are divided into premetallogenic sedimentary diagenesis, metamorphic, hydrothermal metallogenic, and postmetallogenic supergene periods. The Jinping Chang'an gold deposit is divided into sedimentary diagenesis, tectonic-magma-hydrothermal mineralization, and supergene secondary enrichment periods (Figures 6 and 7; Li, 2012; Zhang et al., 2014) [37,41]. Moreover, from detailed field observations, hand specimens, and microscopic observations, the mineral associations of various deposits were determined. The hydrothermal mineralization stage was divided according to the cutting relationship between the mineralized veins and the structural characteristics of the ore. 
Table 1. Geological characteristics of the main gold deposits in Ailaoshan metallogenic belt.

\begin{tabular}{|c|c|c|c|c|}
\hline Gold Deposit & Zhenyuan Laowangzhai & Mojiang Jinchang & Yuanyang Daping & Jinping Chang'an \\
\hline Proven reserves $(\mathrm{t})$ & 22.484 & 31.740 & 60 & 31.155 \\
\hline Average grade $(\mathrm{g} / \mathrm{t})$ & 5.29 & 2.69 & 14.30 & 5.84 \\
\hline Ore-controlling structure & $\begin{array}{l}\text { W-trending Jiujia-Mojiang fault and } \\
\text { EW-trending Bankan river fault } \\
\text { intersection site, the secondary } \\
\text { EW-trending thrust brittle-ductile } \\
\text { fault and its derived interlayer } \\
\text { fault zone }\end{array}$ & $\begin{array}{l}\text { NW-trending NNE-trending } \\
\text { secondary normal fault and } \\
\text { translation fault on the east side of } \\
\text { Jiujia-Mojiang fault }\end{array}$ & $\begin{array}{l}\text { NW to both sides of the Jinzi river } \\
\text { fault, between the Sanjia river fault } \\
\text { and the Xiaozhai-Jinping fault }\end{array}$ & $\begin{array}{l}\text { NW-trending Tongjiao river fault and } \\
\text { Ailaoshan fault intersection site, } \\
\text { secondary NW-trending nappe } \\
\text { structure slip surface and conjugate } \\
\text { NE-trending fault }\end{array}$ \\
\hline Magmatite & $\begin{array}{l}\text { It is dominated by ultrabasic rocks } \\
\text { and basic rocks, followed by } \\
\text { lamprophyre and quartz porphyry }\end{array}$ & The ultrabasic Jinchang rock pluton & $\begin{array}{l}\text { Hercynian Taojiazhai diorite, } \\
\text { Yanshanian monzogranite } \\
\text { and granite }\end{array}$ & $\begin{array}{c}\text { Himalayan olivine pyroxenite, diabase, } \\
\text { gabbro, syenite porphyry, syenite, fine } \\
\text { grained syenite and granite } \\
\text { and lamprophyre }\end{array}$ \\
\hline Host rock & $\begin{array}{l}\text { The Lower Carboniferous altered } \\
\text { basalt and lamprophyre are } \\
\text { dominated, with a small amount of } \\
\text { carbonaceous siliceous slate } \\
\text { and sandstone. }\end{array}$ & $\begin{array}{l}\text { Upper Devonian metamorphic } \\
\text { quartz sandstone, carbon-bearing } \\
\text { slate, silicified sandstone, siliceous } \\
\text { rock and pebbly quartzite }\end{array}$ & Hercynian Taojiazhai diorite & $\begin{array}{l}\text { Middle-Upper Silurian dolomite, Lower } \\
\text { Ordovician siltstone intercalated with } \\
\text { mudstone and conglomerate, and } \\
\text { altered diabase of the Himalayan period }\end{array}$ \\
\hline Mineralization style & $\begin{array}{l}\text { Disseminated, veined, reticulated } \\
\text { and breccia altered rock types }\end{array}$ & $\begin{array}{l}\text { Banded, fine vein disseminated and } \\
\text { breccia quartz vein type and altered } \\
\text { rock type }\end{array}$ & $\begin{array}{l}\text { Veinlets disseminated quartz } \\
\text { vein type }\end{array}$ & $\begin{array}{l}\text { Veined, reticulated and brecciated } \\
\text { altered rock types and quartz vein types }\end{array}$ \\
\hline Morphology of ore body & $\begin{array}{l}\text { The ore bodies produced along the } \\
\text { faults are mainly veins and irregular } \\
\text { veins; the ore bodies produced } \\
\text { along the layers are layered } \\
\text { and lenticular }\end{array}$ & $\begin{array}{l}\text { The ore bodies produced in } \\
\text { quartzite are mostly layered and } \\
\text { lenticular, and the ore bodies } \\
\text { produced in quartz veins are often } \\
\text { arranged in geese and appear } \\
\text { in groups }\end{array}$ & $\begin{array}{l}\text { Thin veins, not far apart and spread } \\
\text { out in parallel }\end{array}$ & Flame shaped, lenticular structure \\
\hline Orebody scale & Length $>1000 \mathrm{~m}$, thickness $>1 \mathrm{~m}$ & $\begin{array}{l}\text { Length }>1000 \mathrm{~m} \text {, width }>100 \mathrm{~m} \text {, the } \\
\text { average thickness is } 16.25 \mathrm{~m}\end{array}$ & Length $>150 \mathrm{~m}$, thickness $>2 \mathrm{~m}$ & $\begin{array}{l}1800 \mathrm{~m} \text { long, }>40 \mathrm{~m} \text { thick, with an } \\
\text { average thickness of } 25 \mathrm{~m}\end{array}$ \\
\hline
\end{tabular}


Table 1. Cont

\begin{tabular}{|c|c|c|c|c|}
\hline Gold Deposit & Zhenyuan Laowangzhai & Mojiang Jinchang & Yuanyang Daping & Jinping Chang'an \\
\hline Ore type & $\begin{array}{l}\text { Mainly mineralized ultrabasic-basic } \\
\text { rocks, sandy (siliceous) sericite and } \\
\text { metamorphic sandstone, and some } \\
\text { are mineralized basalt } \\
\text { and lamprophyre. }\end{array}$ & $\begin{array}{l}\text { Gold-bearing quartz veins, } \\
\text { disseminated gold-bearing } \\
\text { quartzite, and gold-bearing variable } \\
\text { residual siltstone. }\end{array}$ & $\begin{array}{c}\text { Sulfide-quartz vein type, fracture } \\
\text { zone altered rock type and } \\
\text { breccia type. }\end{array}$ & $\begin{array}{l}\text { Veinlets disseminated sulfide ores and } \\
\text { breccia-like oxide ores. }\end{array}$ \\
\hline Gold-bearing mineral & Mainly pyrite & $\begin{array}{l}\text { Mainly pyrite, pyrrhotite and } \\
\text { marcasite }\end{array}$ & Mainly pyrite and galena & Quartz, pyrite, arsenopyrite \\
\hline Ore mineral & $\begin{array}{l}\text { Pyrite, arsenopyrite, arsenopyrite, } \\
\text { stibnite, scheelite, galena, sphalerite, } \\
\text { chalcopyrite, arsenopyrite, etc. }\end{array}$ & $\begin{array}{l}\text { Pyrite, pyrrhotite, white ore, } \\
\text { chalcopyrite, bornite, stibnite, } \\
\text { galena, sphalerite, etc. }\end{array}$ & $\begin{array}{l}\text { Pyrite, galena, chalcopyrite, } \\
\text { sphalerite, wolframite, arsenic } \\
\text { tetrahedrite, etc. }\end{array}$ & Pyrite, arsenopyrite, hematite, etc. \\
\hline Gangue mineral & Quartz, iron dolomite, sericite, etc. & $\begin{array}{l}\text { Quartz, chalcedony, } \\
\text { cryptocrystalline siliceous, opal, } \\
\text { sericite, chlorite, albite, etc. }\end{array}$ & Quartz, iron dolomite, barite, etc. & $\begin{array}{l}\text { Quartz, feldspar, sericite, clay minerals, } \\
\text { Muscovite, dolomite, calcite, etc. }\end{array}$ \\
\hline Occurrence of gold & Natural gold and silver gold mines & $\begin{array}{l}\text { Natural gold, silver gold mine, } \\
\text { selenium gold silver mine, iridium } \\
\text { gold mine and palladium gold mine }\end{array}$ & Natural gold and silver gold mines & Natural gold and silver gold mines \\
\hline Alteration characteristics & $\begin{array}{l}\text { Metasomatic quartzification, } \\
\text { silicification, sericitization, } \\
\text { carbonation and associated } \\
\text { sulfidation }\end{array}$ & $\begin{array}{l}\text { Silicification, pyritization, } \\
\text { chromium hydromica, liquification, } \\
\text { and carbonation }\end{array}$ & $\begin{array}{l}\text { Silicification, pyritization, } \\
\text { sericitization, chloritization, epidote } \\
\text { and carbonation }\end{array}$ & $\begin{array}{l}\text { Silicification, carbonation, sericite, } \\
\text { pyrite, etc. }\end{array}$ \\
\hline References & {$[20,42,43]$} & {$[32,39,44-46]$} & {$[4,21,40,42]$} & {$[16,47-50]$} \\
\hline
\end{tabular}




\begin{tabular}{|c|c|c|c|c|c|c|}
\hline \multirow{2}{*}{ Mineral } & \multirow[b]{2}{*}{ Sedimentation } & \multirow[b]{2}{*}{ Metamorphic } & \multicolumn{3}{|c|}{ Hydrothermal vein } & \multirow[b]{2}{*}{ Supergene } \\
\hline & & & Early stage & Mainly stage & Late stage & \\
\hline Quartz & 可品 & प्व & 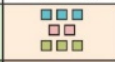 & 㗊 & 㗊 & 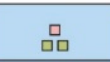 \\
\hline Chalcedony & & & & & व्र & \\
\hline Sericite & & טחם & םם & ם & ם & \\
\hline Calcite & & & & $\begin{array}{l}\text { पवप्र } \\
\text { व० }\end{array}$ & 㗊 & व \\
\hline Millerite & & & वप्व & 口 & & \\
\hline Gersdorffite & & & पष्प & ㅁ & & \\
\hline Scheelite & & & प्व & व & $\square$ & \\
\hline Pyrite & 㗊 & पवा & $\begin{array}{l}\text { 㗊㗊 } \\
\text { ㅁㅁㅁ }\end{array}$ & 㗊品 & 哭 & \\
\hline Copper pyrites & & $\square$ & ㅁㅁㅁㅁㅏ & 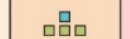 & 口 & \\
\hline Sphalerite & & $\square$ & $\begin{array}{l}\text { वם } \\
\text { वप्र }\end{array}$ & 뭄 & & \\
\hline Chalcostibite & & 口 & ם & 口 & & \\
\hline Ullmannite & & & व & 口 & & \\
\hline Tetrahedrite & & $\square$ & व & $\square$ & & \\
\hline Galena & & 口 & 吅 & هם & 口 & \\
\hline Stibnite & & & & & वप्र & \\
\hline $\begin{array}{l}\text { Chromium } \\
\text { hydromica }\end{array}$ & 口 & & ם & व्र & & \\
\hline Polybasite & & & $\square$ & प्व & & \\
\hline Gold & & & 몸 & 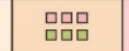 & 品 & \\
\hline Arsenopyrite & & & & प्व & & \\
\hline Magnetite & ם & & & & 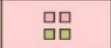 & \\
\hline Limonite & & & & & & $\begin{array}{l}\text { 㗊品 } \\
\text { 㗊 }\end{array}$ \\
\hline
\end{tabular}

Figure 6. Minerals of the Zhenyuan Laowangzhai, Yuanyang Daping, and Mojiang Jinchang gold deposits (modified from [22]).

\begin{tabular}{|c|c|c|c|}
\hline Mineral & Sedimentation & $\begin{array}{c}\text { Hydrothermal } \\
\text { phase }\end{array}$ & $\begin{array}{c}\text { Supergene } \\
\text { phase }\end{array}$ \\
\hline $\begin{array}{c}\text { Franmboidal } \\
\text { pyrite }\end{array}$ & प्व & & \\
\hline $\begin{array}{l}\text { Hydrothermal } \\
\text { pyrite }\end{array}$ & & प्व & \\
\hline Gold & & प्व & \\
\hline Arsenopyrite & & प्व & \\
\hline Chalcopyrite & & वם & \\
\hline Galena & & प्व & \\
\hline Sphalerite & & प् & \\
\hline Magnetite & & $\square$ & \\
\hline $\begin{array}{l}\text { Hydrothermal } \\
\text { quartz }\end{array}$ & & ㅁㅁ & \\
\hline Carbonate & & प्व & \\
\hline Sericite & & $\square$ & \\
\hline Limonite & & & 口प \\
\hline Clay minerals & & & प्व \\
\hline
\end{tabular}

Figure 7. Minerals of the Jinping Chang'an gold deposits (modified from [41]). 


\section{Metallogenic Geodynamic Background}

Yang et al. (2010) and Zhao (2014) [22,36] collected metallogenic age data of four gold deposits in the Ailaoshan metallogenic belt, and this study makes a detailed supplement on this basis (Table 2 and Figure 8). Based on the characteristics of age data, the metallogenic ages of the four main gold deposits in the metallogenic belt are concentrated in the Himalayan period, corresponding to the India-Eurasia continental collision period. It might have experienced early (65-60 Ma), major (40-30 Ma), and late (30-25 Ma) mineralization-thermal events. Wang et al. (2005) [51] proposed that the three episodes of gold mineralization are associated with three events of tectonic-magmatism and/or fluid events. Even though the gold deposits were formed at different episodes, all of the deposits are genetically related to the tectonic movements in large-scale shear zones. The tectonic events (including large-scale strike-slip) among the Paleogene considerably affected gold mineralization, with new deposits formed and old deposits enriched or superimposed to be a higher grade by a new mineralization stage. The data indicate that gold deposits were concentrated in some areas and were mostly formed at different period boundaries, indicating that some peak stages of gold mineralization (metallogenic episodes) existed and gold deposits were mostly formed by episodic mineralization [51,52]. However, since these mineralization ages are obtained by applying different dating methods to minerals that are not directly related to gold mineralization, the reliability of the data must be verified (Yang et al., 2011) [23].

Table 2. Mineralization and magmatic age of the main gold deposits in Ailaoshan metallogenic belt.

\begin{tabular}{|c|c|c|c|c|c|c|c|}
\hline No. & Deposit & Sample No. & $\begin{array}{c}\text { Sample } \\
\text { Description }\end{array}$ & Mineral & $\begin{array}{l}\text { Dating } \\
\text { Methods }\end{array}$ & Age/Ma & References \\
\hline 1 & $\begin{array}{l}\text { Mojiang } \\
\text { Jinchang }\end{array}$ & L3 & Nickel ore & $\begin{array}{l}\text { Chromium } \\
\text { sericite }\end{array}$ & ${ }^{40} \mathrm{Ar}-{ }^{39} \mathrm{Ar}$ & $63.09 \pm 0.16$ & [33] \\
\hline 2 & $\begin{array}{l}\text { Mojiang } \\
\text { Jinchang }\end{array}$ & L5 & Nickel ore & $\begin{array}{l}\text { Chromium } \\
\text { sericite }\end{array}$ & ${ }^{40} \mathrm{Ar}^{39} \mathrm{Ar}$ & $62.05 \pm 0.14$ & [33] \\
\hline 3 & $\begin{array}{l}\text { Mojiang- } \\
\text { Jinchang }\end{array}$ & Sh4 & Nickel ore & $\begin{array}{l}\text { Chromium } \\
\text { sericite }\end{array}$ & ${ }^{40} \mathrm{Ar}-{ }^{39} \mathrm{Ar}$ & $61.55 \pm 0.23$ & [33] \\
\hline 4 & $\begin{array}{l}\text { Mojiang } \\
\text { Jinchang }\end{array}$ & & Gold ore & $\begin{array}{l}\text { Chromium } \\
\text { hydromica }\end{array}$ & ${ }^{40} \mathrm{~K}-{ }^{40} \mathrm{Ar}$ & 61 & [53] \\
\hline 5 & $\begin{array}{l}\text { Mojiang } \\
\text { Jinchang }\end{array}$ & 9704 & $\begin{array}{l}\text { Auriferous } \\
\text { quartz vein }\end{array}$ & Quartz & ${ }^{40} \mathrm{Ar}-{ }^{39} \mathrm{Ar}$ & $56.49 \pm 0.34$ & [33] \\
\hline 6 & $\begin{array}{l}\text { Mojiang } \\
\text { Jinchang }\end{array}$ & M4 & $\begin{array}{l}\text { Auriferous } \\
\text { quartz vein }\end{array}$ & Quartz & ${ }^{40} \mathrm{Ar}-{ }^{39} \mathrm{Ar}$ & $59.67 \pm 0.16$ & [33] \\
\hline 7 & $\begin{array}{l}\text { Mojiang } \\
\text { Jinchang }\end{array}$ & Sh2-a & $\begin{array}{l}\text { Auriferous } \\
\text { quartz vein }\end{array}$ & Quartz & ${ }^{40} \mathrm{Ar}-{ }^{39} \mathrm{Ar}$ & $54.02 \pm 0.19$ & [33] \\
\hline 8 & $\begin{array}{l}\text { Mojiang } \\
\text { Jinchang }\end{array}$ & Sh2-c & $\begin{array}{l}\text { Auriferous } \\
\text { quartz vein }\end{array}$ & Quartz & ${ }^{40} \mathrm{Ar}-{ }^{39} \mathrm{Ar}$ & $55.74 \pm 0.34$ & [33] \\
\hline 9 & $\begin{array}{l}\text { Mojiang } \\
\text { Jinchang }\end{array}$ & HBM-62 & $\begin{array}{l}\text { Auriferous } \\
\text { quartz vein }\end{array}$ & Quartz & ESR & 66.4 & [54] \\
\hline 10 & $\begin{array}{l}\text { Mojiang } \\
\text { Jinchang }\end{array}$ & HBM-63 & $\begin{array}{l}\text { Auriferous } \\
\text { quartz vein }\end{array}$ & Quartz & ESR & 29 & [54] \\
\hline 11 & $\begin{array}{l}\text { Mojiang } \\
\text { Jinchang }\end{array}$ & HBM-105 & $\begin{array}{l}\text { Auriferous } \\
\text { quartz vein }\end{array}$ & Quartz & ESR & 44.8 & {$[54]$} \\
\hline 12 & $\begin{array}{l}\text { Zhenyuan } \\
\text { Laowangzhai }\end{array}$ & & Lamprophyre & Phlogopite & ${ }^{87} \mathrm{Rb}-{ }^{86} \mathrm{Sr}$ & 35.6 & [53] \\
\hline 13 & $\begin{array}{l}\text { Zhenyuan } \\
\text { Laowangzhai }\end{array}$ & & Lamprophyre & Phlogopite & ${ }^{87} \mathrm{Rb}-{ }^{86} \mathrm{Sr}$ & 43 & [53] \\
\hline 14 & $\begin{array}{l}\text { Zhenyuan } \\
\text { Laowangzhai }\end{array}$ & & Minette & Apatite & F-T & $22.7 \pm 3.3$ & [38] \\
\hline 15 & $\begin{array}{l}\text { Zhenyuan } \\
\text { Laowangzhai }\end{array}$ & & Minette & Apatite & F-T & $27.1 \pm 2.8$ & [38] \\
\hline 16 & $\begin{array}{l}\text { Zhenyuan } \\
\text { Laowangzhai }\end{array}$ & LWZ-1 & Lamprophyre & Phlogopite & ${ }^{40} \mathrm{Ar}-{ }^{39} \mathrm{Ar}$ & $30.8 \pm 0.4$ & [55] \\
\hline
\end{tabular}


Table 2. Cont.

\begin{tabular}{|c|c|c|c|c|c|c|c|}
\hline No. & Deposit & Sample No. & $\begin{array}{c}\text { Sample } \\
\text { Description }\end{array}$ & Mineral & $\begin{array}{l}\text { Dating } \\
\text { Methods }\end{array}$ & Age/Ma & References \\
\hline 17 & $\begin{array}{l}\text { Zhenyuan } \\
\text { Laowangzhai }\end{array}$ & LWZ-2 & Lamprophyre & Phlogopite & ${ }^{40} \mathrm{Ar}-{ }^{39} \mathrm{Ar}$ & $34.3 \pm 0.2$ & [55] \\
\hline 18 & $\begin{array}{l}\text { Zhenyuan } \\
\text { Laowangzhai }\end{array}$ & & $\begin{array}{l}\text { Mineralized } \\
\text { lamprophyre }\end{array}$ & Total rock & ${ }^{87} \mathrm{Rb}^{86} \mathrm{Sr}$ & 28.2 & [56] \\
\hline 19 & $\begin{array}{l}\text { Zhenyuan } \\
\text { Laowangzhai }\end{array}$ & & $\begin{array}{l}\text { Mineralized } \\
\text { lamprophyre }\end{array}$ & Phlogopite & ${ }^{40} \mathrm{Ar}-{ }^{39} \mathrm{Ar}$ & $26.4 \pm 0.2$ & [55] \\
\hline 20 & $\begin{array}{l}\text { Zhenyuan } \\
\text { Laowangzhai }\end{array}$ & & $\begin{array}{l}\text { Mineralized } \\
\text { ultrabasic } \\
\text { rocks }\end{array}$ & $\begin{array}{l}\text { Chromium } \\
\text { hydromica }\end{array}$ & ${ }^{87} \mathrm{Rb}-{ }^{86} \mathrm{Sr}$ & 32.8 & [56] \\
\hline 21 & $\begin{array}{l}\text { Zhenyuan } \\
\text { Laowangzhai }\end{array}$ & & $\begin{array}{l}\text { Mineralized } \\
\text { ultrabasic } \\
\text { rocks }\end{array}$ & $\begin{array}{l}\text { Chromium } \\
\text { hydromica }\end{array}$ & ${ }^{87} \mathrm{Rb}^{86} \mathrm{Sr}$ & 39.6 & [56] \\
\hline 22 & $\begin{array}{l}\text { Zhenyuan } \\
\text { Laowangzhai }\end{array}$ & & $\begin{array}{l}\text { Altered } \\
\text { minette }\end{array}$ & Biotite & ${ }^{87} \mathrm{Rb}^{86} \mathrm{Sr}$ & 49.3 & [57] \\
\hline 23 & $\begin{array}{l}\text { Zhenyuan } \\
\text { Laowangzhai }\end{array}$ & L-48 & $\begin{array}{l}\text { Auriferous } \\
\text { quartz vein }\end{array}$ & Quartz & ESR & 37.9 & [54] \\
\hline 24 & $\begin{array}{l}\text { Zhenyuan } \\
\text { Laowangzhai }\end{array}$ & L-12 & $\begin{array}{l}\text { Auriferous } \\
\text { quartz vein }\end{array}$ & Quartz & ESR & 54.2 & [54] \\
\hline 25 & $\begin{array}{l}\text { Zhenyuan } \\
\text { Laowangzhai }\end{array}$ & W-26 & $\begin{array}{l}\text { Auriferous } \\
\text { quartz vein }\end{array}$ & Quartz & ESR & 47.4 & [54] \\
\hline 26 & $\begin{array}{l}\text { Zhenyuan } \\
\text { Laowangzhai }\end{array}$ & & & Sericite & ${ }^{40} \mathrm{~K}-{ }^{40} \mathrm{Ar}$ & 50 & [58] \\
\hline 27 & $\begin{array}{l}\text { Zhenyuan } \\
\text { Laowangzhai }\end{array}$ & C02-11 & $\begin{array}{c}\text { Quartz } \\
\text { porphyry }\end{array}$ & Zircon & SHRIMP & $248 \pm 1$ & [59] \\
\hline 28 & $\begin{array}{l}\text { Zhenyuan } \\
\text { Laowangzhai }\end{array}$ & C02-11 & $\begin{array}{l}\text { Quartz } \\
\text { porphyry }\end{array}$ & Zircon & LA-ICP-MS & $247.7 \pm 1.7$ & [59] \\
\hline 29 & $\begin{array}{l}\text { Zhenyuan } \\
\text { Laowangzhai }\end{array}$ & C03-19 & $\begin{array}{l}\text { Quartz } \\
\text { porphyry }\end{array}$ & Zircon & LA-ICP-MS & $255.1 \pm 2$ & [59] \\
\hline 30 & $\begin{array}{l}\text { Zhenyuan } \\
\text { Donggualin }\end{array}$ & & Lamprophyre & Phlogopite & ${ }^{40} \mathrm{Ar}-{ }^{39} \mathrm{Ar}$ & 36.1 & [53] \\
\hline 31 & $\begin{array}{l}\text { Zhenyuan } \\
\text { Donggualin }\end{array}$ & & $\begin{array}{l}\text { Auriferous } \\
\text { quartz vein }\end{array}$ & Quartz & ${ }^{40} \mathrm{Ar}-{ }^{39} \mathrm{Ar}$ & $51.8 \pm 1.7$ & [58] \\
\hline 32 & $\begin{array}{l}\text { Zhenyuan } \\
\text { Donggualin }\end{array}$ & & $\begin{array}{l}\text { Altered } \\
\text { mineralized } \\
\text { rock }\end{array}$ & Sericite & ${ }^{87} \mathrm{Rb}-{ }^{86} \mathrm{Sr}$ & 48.9 & [57] \\
\hline 33 & $\begin{array}{l}\text { Zhenyuan } \\
\text { Kudumu }\end{array}$ & & $\begin{array}{c}\text { Alkaline } \\
\text { lamprophyre }\end{array}$ & Total rock & ${ }^{87} \mathrm{Rb}^{-86} \mathrm{Sr}$ & 29.1 & {$[60]$} \\
\hline 34 & $\begin{array}{l}\text { Zhenyuan } \\
\text { Kudumu }\end{array}$ & & $\begin{array}{l}\text { Altered } \\
\text { mineralized } \\
\text { rock }\end{array}$ & $\begin{array}{l}\text { Chromium } \\
\text { hydromica }\end{array}$ & ${ }^{87} \mathrm{Rb}-{ }^{86} \mathrm{Sr}$ & 64.2 & [57] \\
\hline 35 & $\begin{array}{l}\text { Zhenyuan } \\
\text { Kudumu }\end{array}$ & & $\begin{array}{c}\text { Altered } \\
\text { mineralized } \\
\text { rock }\end{array}$ & Sericite & ${ }^{87} \mathrm{Rb}-{ }^{86} \mathrm{Sr}$ & 61.1 & [57] \\
\hline 36 & $\begin{array}{l}\text { Zhenyuan } \\
\text { Langnitang }\end{array}$ & & Lamprophyre & Phlogopite & ${ }^{40} \mathrm{~K}-{ }^{40} \mathrm{Ar}$ & $30.39 \pm 0.46$ & [53] \\
\hline 37 & $\begin{array}{l}\text { Zhenyuan } \\
\text { Langnitang }\end{array}$ & & Lamprophyre & Phlogopite & ${ }^{40} \mathrm{~K}-{ }^{40} \mathrm{Ar}$ & $30.95 \pm 0.46$ & [53] \\
\hline 38 & $\begin{array}{l}\text { Zhenyuan } \\
\text { Xiachahe }\end{array}$ & & $\begin{array}{l}\text { Auriferous } \\
\text { quartz vein, } \\
\text { mylonite, hy- } \\
\text { permylonite }\end{array}$ & $\begin{array}{l}\text { Hydrothermal } \\
\text { Phlogopite }\end{array}$ & ${ }^{40} \mathrm{Ar}-{ }^{39} \mathrm{Ar}$ & $43.68 \pm 0.32$ & [61] \\
\hline 39 & $\begin{array}{l}\text { Yuanyang } \\
\text { Daping }\end{array}$ & HBD-42 & $\begin{array}{l}\text { Auriferous } \\
\text { quartz vein }\end{array}$ & Quartz & ESR & 58 & [54] \\
\hline 40 & $\begin{array}{l}\text { Yuanyang } \\
\text { Daping }\end{array}$ & HBD-20 & $\begin{array}{l}\text { Auriferous } \\
\text { quartz vein }\end{array}$ & Quartz & ESR & 41.3 & [54] \\
\hline 41 & $\begin{array}{l}\text { Yuanyang } \\
\text { Daping }\end{array}$ & HBD-27 & $\begin{array}{l}\text { Auriferous } \\
\text { quartz vein }\end{array}$ & Quartz & ESR & 48.3 & [54] \\
\hline
\end{tabular}


Table 2. Cont.

\begin{tabular}{|c|c|c|c|c|c|c|c|}
\hline No. & Deposit & Sample No. & $\begin{array}{c}\text { Sample } \\
\text { Description }\end{array}$ & Mineral & $\begin{array}{l}\text { Dating } \\
\text { Methods }\end{array}$ & Age/Ma & References \\
\hline 42 & $\begin{array}{l}\text { Yuanyang } \\
\text { Daping }\end{array}$ & & $\begin{array}{l}\text { Sericitization } \\
\text { diorite }\end{array}$ & Sericite & ${ }^{40} \mathrm{Ar}-{ }^{39} \mathrm{Ar}$ & $33.76 \pm 0.65$ & [62] \\
\hline 43 & $\begin{array}{l}\text { Yuanyang } \\
\text { Daping }\end{array}$ & & Lamprophyre & & ${ }^{40} \mathrm{~K}-{ }^{40} \mathrm{Ar}$ & 49.38 & [53] \\
\hline 44 & $\begin{array}{l}\text { Yuanyang } \\
\text { Daping }\end{array}$ & & $\begin{array}{l}\text { Altered } \\
\text { tectonite }\end{array}$ & Sericite & ${ }^{40} \mathrm{Ar}-{ }^{39} \mathrm{Ar}$ & $33.76 \pm 0.65$ & [62] \\
\hline 45 & $\begin{array}{l}\text { Yuanyang } \\
\text { Daping }\end{array}$ & & Diorite & Zircon & SHRIMP & $773 \pm 12$ & [63] \\
\hline 46 & $\begin{array}{l}\text { Yuanyang } \\
\text { Daping }\end{array}$ & & Diorite & Zircon & LA-ICP-MS & $761.6 \pm 7.4$ & [47] \\
\hline 47 & $\begin{array}{l}\text { Yuanyang } \\
\text { Daping }\end{array}$ & & $\begin{array}{l}\text { Biotite } \\
\text { monzonitic } \\
\text { granite }\end{array}$ & Zircon & LA-ICP-MS & $731 \pm 18$ & [47] \\
\hline 48 & $\begin{array}{l}\text { Jinping } \\
\text { Chang'an }\end{array}$ & & $\begin{array}{l}\text { Altered } \\
\text { quartz } \\
\text { syenite } \\
\text { porphyry }\end{array}$ & Total rock & ${ }^{40} \mathrm{~K}-{ }^{40} \mathrm{Ar}$ & 36 & [64] \\
\hline 49 & $\begin{array}{l}\text { Jinping } \\
\text { Chang'an }\end{array}$ & & $\begin{array}{l}\text { Premineralizatior } \\
\text { hornblende } \\
\text { syenite } \\
\text { porphyry }\end{array}$ & Biotite & ${ }^{87} \mathrm{Rb}-{ }^{86} \mathrm{Sr}$ & 36 & [65] \\
\hline 50 & $\begin{array}{l}\text { Jinping } \\
\text { Chang'an }\end{array}$ & CAl7 & Lamprophyre & Biotite & ${ }^{40} \mathrm{Ar}-{ }^{39} \mathrm{Ar}$ & $35.62 \pm 0.16$ & {$[66]$} \\
\hline 51 & $\begin{array}{l}\text { Jinping } \\
\text { Chang'an }\end{array}$ & & Lamprophyre & Biotite & ${ }^{40} \mathrm{Ar}-{ }^{39} \mathrm{Ar}$ & $35.27 \pm 0.74$ & [66] \\
\hline 52 & $\begin{array}{l}\text { Jinping } \\
\text { Chang'an }\end{array}$ & & Mo ore & Molybdenite & Re-Os & $34.54 \pm 0.69$ & [67] \\
\hline 53 & $\begin{array}{l}\text { Jinping } \\
\text { Chang'an }\end{array}$ & CA11P32 & $\begin{array}{l}\text { Syenite } \\
\text { porphyry }\end{array}$ & Zircon & LA-ICP-MS & $34.49 \pm 0.14$ & [68] \\
\hline 54 & $\begin{array}{l}\text { Jinping } \\
\text { Chang'an }\end{array}$ & CA11P115 & $\begin{array}{l}\text { Syenite } \\
\text { porphyry }\end{array}$ & Zircon & LA-ICP-MS & $34.47 \pm 0.07$ & [68] \\
\hline 55 & $\begin{array}{l}\text { Jinping } \\
\text { Chang'an }\end{array}$ & CAC07 & $\begin{array}{l}\text { Biotite } \\
\text { granite } \\
\text { porphyry }\end{array}$ & Zircon & LA-ICP-MS & $33.66 \pm 0.06$ & [68] \\
\hline 56 & $\begin{array}{l}\text { Jinping } \\
\text { Chang'an }\end{array}$ & CAC09 & $\begin{array}{l}\text { Biotite } \\
\text { granite } \\
\text { porphyry }\end{array}$ & Zircon & LA-ICP-MS & $34.30 \pm 0.05$ & [68] \\
\hline 57 & $\begin{array}{l}\text { Jinping } \\
\text { Tongchang }\end{array}$ & & $\begin{array}{l}\text { Aplite } \\
\text { syenite }\end{array}$ & Zircon & LA-ICP-MS & $36.0 \pm 0.19$ & [69] \\
\hline 58 & $\begin{array}{l}\text { Jinping } \\
\text { Tongchang }\end{array}$ & & Orthophyre & Zircon & LA-ICP-MS & $34.6 \pm 0.2$ & [69] \\
\hline 59 & $\begin{array}{l}\text { Jinping } \\
\text { Tongchang }\end{array}$ & & $\begin{array}{l}\text { Porphyry } \\
\text { copper- } \\
\text { molybdenum } \\
\text { ore }\end{array}$ & Molybdenite & Re-Os & $34.38 \pm 0.46$ & [52] \\
\hline 60 & $\begin{array}{l}\text { Jinping } \\
\text { Tongchang }\end{array}$ & & Mo ore & Molybdenite & Re-Os & $34.04 \pm 0.54$ & [67] \\
\hline 61 & $\begin{array}{l}\text { Jinping } \\
\text { Tongchang }\end{array}$ & & $\begin{array}{l}\text { Amphibole } \\
\text { syenite } \\
\text { porphyry }\end{array}$ & Biotite & ${ }^{40} \mathrm{~K}-{ }^{40} \mathrm{Ar}$ & 36 & [70] \\
\hline 62 & $\begin{array}{l}\text { Jinping } \\
\text { Tongchang }\end{array}$ & X30-13 & $\begin{array}{l}\text { Quartz } \\
\text { syenite } \\
\text { porphyry }\end{array}$ & Whole rock & ${ }^{87} \mathrm{Rb}^{8}{ }^{86} \mathrm{Sr}$ & 33.9 & [52] \\
\hline 63 & $\begin{array}{l}\text { Jinping } \\
\text { Tongchang }\end{array}$ & X30-14 & $\begin{array}{l}\text { Quartz } \\
\text { syenite } \\
\text { porphyry }\end{array}$ & Whole rock & ${ }^{87} \mathrm{Rb}-{ }^{86} \mathrm{Sr}$ & 35.9 & [52] \\
\hline
\end{tabular}


Table 2. Cont.

\begin{tabular}{|c|c|c|c|c|c|c|c|}
\hline No. & Deposit & Sample No. & $\begin{array}{c}\text { Sample } \\
\text { Description }\end{array}$ & Mineral & $\begin{array}{l}\text { Dating } \\
\text { Methods }\end{array}$ & Age/Ma & References \\
\hline 64 & $\begin{array}{l}\text { Jinping } \\
\text { Tongchang }\end{array}$ & X30-22 & $\begin{array}{c}\text { Quartz } \\
\text { syenite } \\
\text { porphyry }\end{array}$ & Whole rock & ${ }^{87} \mathrm{Rb}-{ }^{86} \mathrm{Sr}$ & 36.1 & [52] \\
\hline 65 & $\begin{array}{c}\text { Jinping } \\
\text { Tongchang }\end{array}$ & & $\begin{array}{l}\text { Syenite } \\
\text { porphyry }\end{array}$ & Biotite & ${ }^{40} \mathrm{~K}-{ }^{40} \mathrm{Ar}$ & $37.7 \pm 0.7$ & [70] \\
\hline 66 & $\begin{array}{c}\text { Jinping } \\
\text { Tongchang }\end{array}$ & TC78 & $\begin{array}{c}\text { Monzogranite } \\
\text { porphyry }\end{array}$ & Zircon & LA-ICP-MS & $35.1 \pm 0.3$ & [71] \\
\hline
\end{tabular}
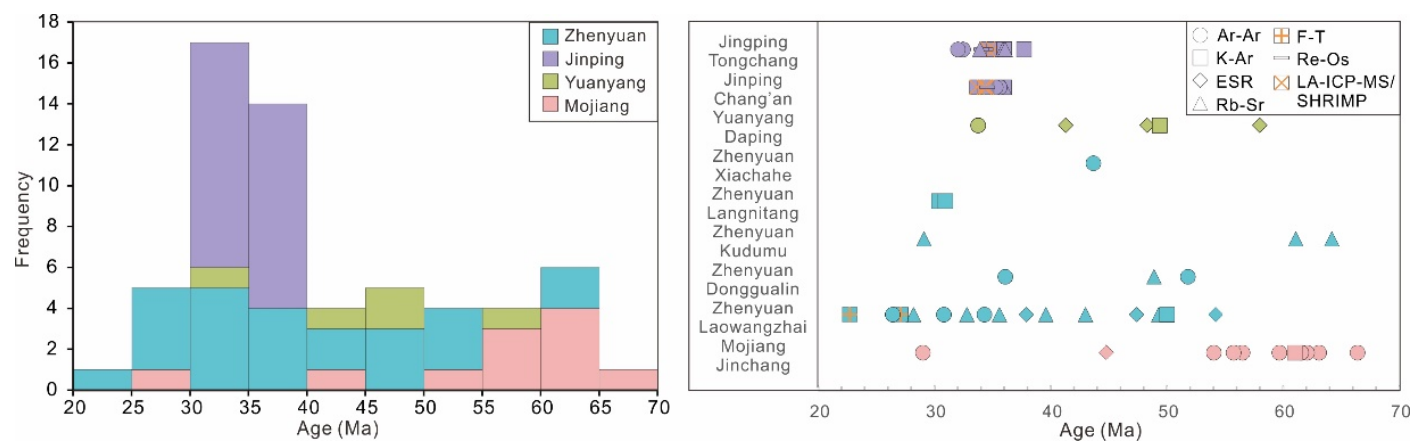

Figure 8. Age histograms and scatter plots of gold deposits and related magmatic activities in the Ailaoshan metallogenic belt (data from Table 2; Ar-Ar: using the step-heating ${ }^{40} \mathrm{Ar}-{ }^{39} \mathrm{Ar}$ method; $\mathrm{K}-\mathrm{Ar}:{ }^{40} \mathrm{~K}-{ }^{40} \mathrm{Ar}$ isochron dating method; Electron Spin Resonance (ESR) dating; Rb-Sr: ${ }^{87} \mathrm{Rb}-{ }^{86} \mathrm{Sr}$ isochron dating method; fission track (F-T) dating method; Laser Ablation Inductively Coupled Plasma-Mass Spectrometry (LA-ICP-MS) zircon U-Pb dating; SHRIMP: dating by single grain zircon ion probe mass spectrometry; Re-Os: ${ }^{187} \mathrm{Re}^{187}$ Os isotope dating method).

Beginning at $65 \mathrm{Ma}$, the India-Eurasia continental collision occurred under the subduction of the Neo-Tethys oceanic crust and the entire Tethys tectonic framework was strongly modified, making Ailaoshan a lateral material adjustment zone for continental collision. The Ailaoshan metallogenic belt has become a structural transformation domain, absorbing and adjusting the strain generated in the collision process (Deng et al., 2011) [72]. Due to the rapid increase in the plate convergence rate, the rocks in the Ailaoshan area have been thrust and faulted due to powerful compression, forming a large-scale thrust-nappe tectonic system. The simultaneous formation of shear strike-slip fault structures (Yang et al., 2011) [23] has significantly shortened and thickened the crust and lithosphere in western Yunnan (Chen et al., 2008) [73]. Simultaneously, along with the deep melting of landing crust, crust-mantle action, and mantle-derived magmatic activity, the tectonic framework and material basis of the Ailaoshan metallogenic belt are laid. The gold mineralization age data show that some gold mineralization occurred during the 65-50 Ma period (Figure 9, Yang et al., 2011) [23].

The main periods of gold mineralization in the Ailaoshan area occurred during 50-30 Ma. During this period, the compression stress concentration between the blocks exceeded the limit. Due to the limitation of boundary conditions, some materials and blocks were extruded to the southeast, resulting in the large-scale strike-slip shear zone of Ailaoshan. At approximately $40 \mathrm{Ma}$, the relative compression rate between India and the Yangtze block decreased significantly. Simultaneously, the direction of mutual movements between them gradually changed, which manifested as a clockwise rotation, and the regional tectonic system moved from compression to the early stage of stretch conversion (Figure 9, Yang et al., 2011) [23]. With decreasing regional compression stress, several strike-slip shear faults and alkali-rich porphyries developed in the Ailaoshan area in the early stage of tectonic regime transformation, indicating that they were uniformly controlled by the tectonic dynamic transformation system of the collision between India and 
the Yangtze block. The large-scale mineralization of gold in the region is closely related to the transformation of the tectonic regime and the strong interaction of the crust and mantle (Yang et al., 2011) [23].

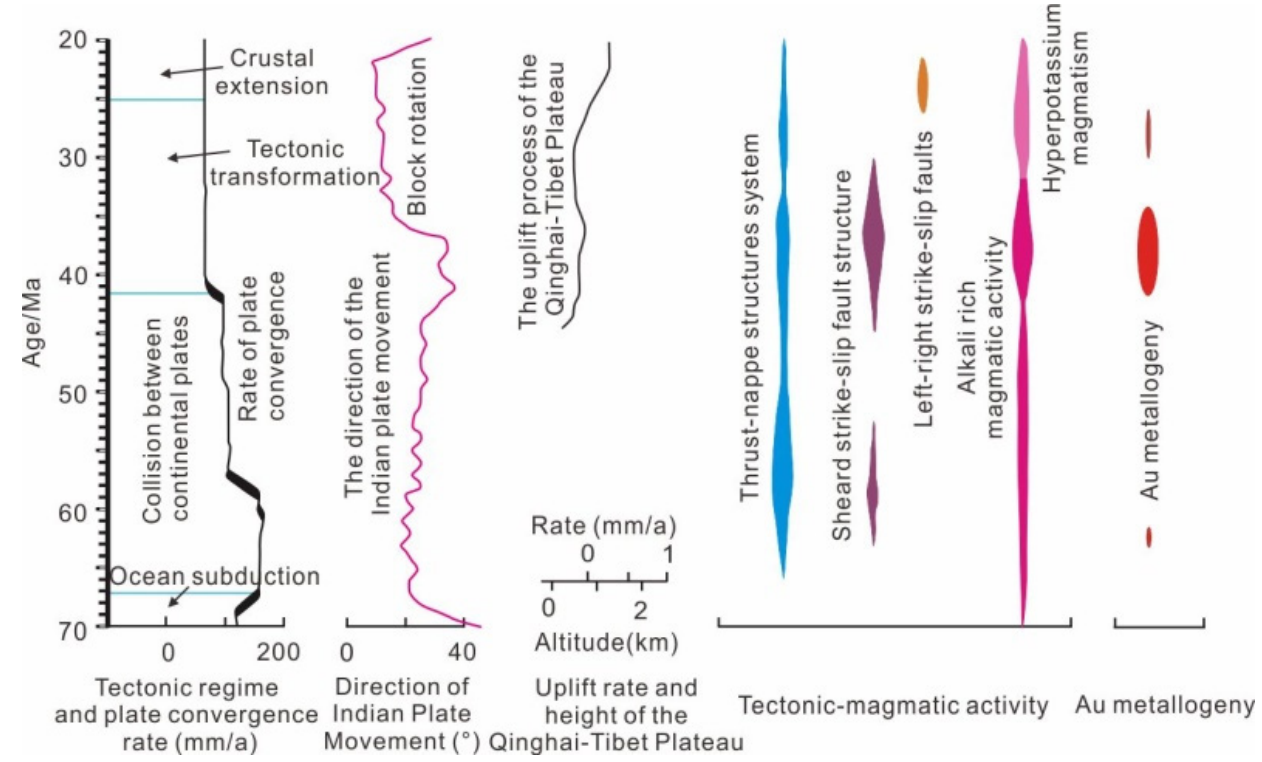

Figure 9. Tectonic-metallogenic evolution sequence of the Ailaoshan metallogenic belt (modified from [23]).

After $30 \mathrm{Ma}$, the gold mineralization in the Ailaoshan area ended and only sporadic age records exist in the Laowangzhai mining area. During this period, the regional tectonic dynamic regime was in the late transition period, the Indian plate changed from a counterclockwise rotation in the early period to a clockwise rotation (Funahara et al., 1992, 1993; Huang and Opdyke, 1993; Chen et al., 1995; Sato et al., 1999) [74-78], and the regional thrust-nappe tectonic system and shear strike-slip fault activities ended (Figure 9 , Yang et al., 2011) [23].

\section{Genesis Mechanism of Ore Deposits}

This paper summarizes the microthermometric data results and $\mathrm{C}-\mathrm{H}-\mathrm{O}-\mathrm{S}-\mathrm{Pb}$ isotopes values of fluid inclusions of four main large gold deposits in the Ailaoshan metallogenic belt, and analyzes the geochemical characteristics and stable isotopes of the ore-forming fluid [16,20,21,42,44-49,53,54,68,79-112] (Li, 2014; Hu et al., 1995; Li et al., 1998; Deng et al., 2013a, b, c; 2015a, b; Mo et al., 2009; Huang et al., 1996; 1998; Zhang et al., 2010; Zhao et al., 2013; Zhang et al., 2019; Qu, 2019; Yang et al., 2013; Li, 1992; 1998; Zhang et al., 1987; Xie et al., 2001; Xiong, 2014; Bi et al., 1996; Zhang et al., 1984; Yang, 2018; Zhu et al., 2011; Chen et al., 2015; Shi et al., 2010; Li, 2017; Han et al., 1997; Li et al., 2000; Ge et al., 2007; Yuan et al., 2010, 2011; Yang et al., 2013; Chang et al., 2005; Zhang, 2017; Ying et al., 2006; Chen, 2010; Li et al., 2011; Fan et al., 2010; Sun et al., 2009; Li et al., 2000; Yang et al., 2021; Zhang et al., 2014; 2017; Li et al., 2013a, b; ECUT, 2007; Zhang et al., 2018).

\subsection{Fluid Inclusion}

The fluid inclusion composition in the Jinping Chang'an, Zhenyuan Laowangzhai, Mojiang Jinchang, and Yuanyang Daping gold deposits are mostly $\mathrm{H}_{2} \mathrm{O}$ and $\mathrm{CO}_{2}(\mathrm{Bi}$ et al., 1997; Xie et al., 2001; Chen et al., 2010; Zhao, 2014) [22,92,107,113] and contain a small amount of $\mathrm{CH}_{4}$. The Chang'an gold deposit also contains a small amount of $\mathrm{C}_{4} \mathrm{H}_{6}(\mathrm{Chen}$ et al., 2010) [107], and the Daping gold deposit contains a small amount of $\mathrm{C}_{2} \mathrm{H}_{4}$ and $\mathrm{N}_{2}$ $(\mathrm{Du}, 2015)$ [114]. Although their compositions are similar, the temperature and pressure of different deposits differ. The fluids of the main mineralization periods typically have the characteristics of medium-low temperatures and salinity (Figure 10), among which 
the mineralization depth is in the order of Yuanyang Daping $>$ Zhenyuan Laowangzhai $>$ Mojiang Jinchang > Jinping Chang'an. Ore-forming fluids are all reducing fluids, and gold might migrate in the form of $\mathrm{Au}(\mathrm{HS})^{2-}$. Table 3 summarizes the petrographic, physical, and chemical parameters of fluid inclusions in the four main gold deposits.

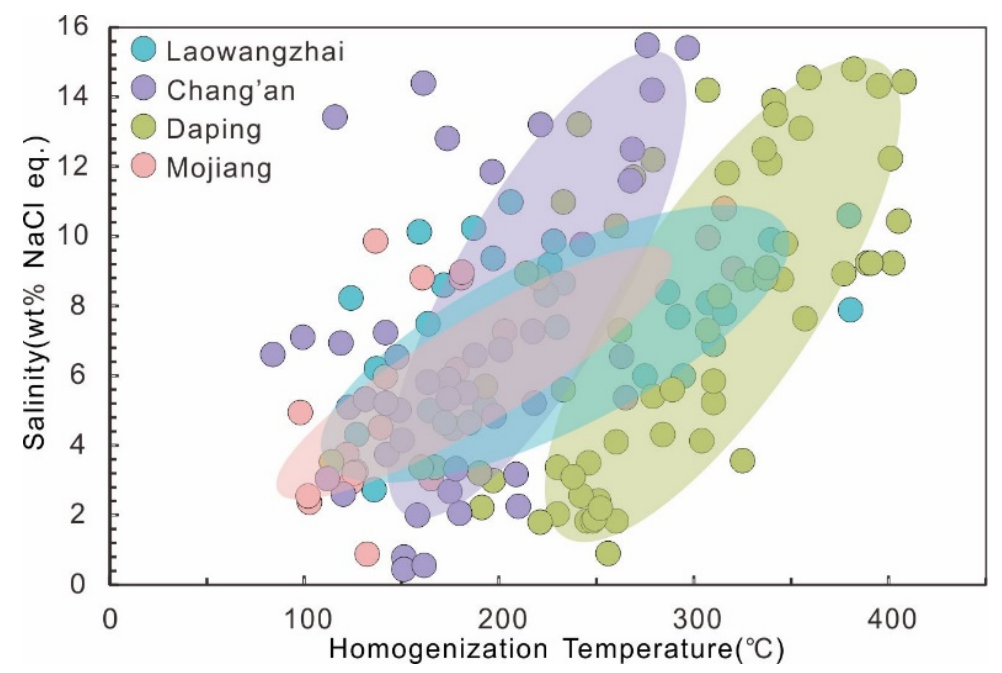

Figure 10. Homogenization Temperature-Salinity map of ore-forming fluid in the Ailaoshan gold deposit. Note: The different colored ellipses indicate the main scope of "Homogenization TemperatureSalinity" in different deposits.

\subsection{H-O Isotopes}

The $\delta \mathrm{D}$ value in the four main gold deposits varies significantly, and the ore-forming fluid sources of different gold deposits are slightly different. Previous studies have shown that the ore-forming fluids of the Zhenyuan Laowangzhai and Yuanyang Daping gold deposits may mostly originate from the mixing of deep-source mantle-derived and metamorphic fluids (Sun et al., 2009; Deng et al., 2013c; Li, 2014; Chen et al., 2014; Du, 2015; Yang, 2018; Xin, 2019; Zhang et al., 2019) [20,21,39,40,82,88,115,116]. Among them, the early Daping ore-forming fluids mostly originated from metamorphic fluids (metamorphic water). The late ore-forming fluid composition is close to atmospheric precipitation (Sun et al., 2009; Zhang et al., 2018) [21,42]. The ore-forming fluids of the Mojiang Jinchang gold-nickel deposit might be deep fluids (mantle-derived or deep metamorphic fluids), with the obvious addition of atmospheric precipitation (Li, 1992; Fan et al., 2010; Xiong et al., 2015; Yang, 2018; Qu, 2019) [32,39,44-46]. The ore-forming fluid of the Jinping Chang'an gold deposit has obvious crust-derived characteristics (higher $\delta^{18} \mathrm{O}_{\mathrm{V}-\mathrm{SMOW}}$ value), and the main body is a metamorphic fluid, which might be mixed with magmatic fluids and atmospheric precipitation (Deng et al., 2012) [117].

This study found that among the four gold deposits in the Ailaoshan metallogenic belt, only the $\delta^{18} \mathrm{O}_{\mathrm{V}-\mathrm{SMOW}}$ values of fluid inclusions in the Zhenyuan Laowangzhai gold deposit and Yuanyang Daping gold deposit are within the $\delta^{18} \mathrm{O}_{\mathrm{V}-\mathrm{SMOW}}$ value range of the generalized magmatic fluid (5.5-10\% [118], Taylor, 1997, Figures 11a and 12d,e). At the same time, their $\delta \mathrm{D}$ values are also in the $\delta \mathrm{D}$ value range of magmatic fluid $(-85 \%$ $-50 \%$ [118], Taylor, 1997, Figures 11a and 12d,e). The Mojiang Jinchang gold deposit and the Jinping Chang'an gold deposit have only a few points located in these two areas, and the degasification of magmatic-hydrothermal fluids should lead to a decrease in the $\delta \mathrm{D}$ value (Taylor et al., 1983) [119]. Therefore, in Figure 11a, all points tend to be close to organic water area. 
Table 3. Physical and chemical conditions of ore-forming fluids in main gold deposits in Ailaoshan metallogenic belt.

\begin{tabular}{|c|c|c|c|c|}
\hline Gold Deposit & Zhenyuan Laowangzhai & Mojiang Jinchang & Yuanyang Daping & Jinping Chang'an \\
\hline Fluid inclusion & $\begin{array}{l}\text { It is mainly composed of gas-liquid } \\
\text { two-phase aqueous solution } \\
\text { inclusions, } \mathrm{CO}_{2} \sim \mathrm{H}_{2} \mathrm{O} \text { three-phase } \\
\text { inclusions and two-phase } \mathrm{CO}_{2} \\
\text { inclusions, with a gas-liquid ratio of } \\
10 \% \text { to } 60 \%\end{array}$ & $\begin{array}{l}\text { Mainly gas-liquid and pure liquid } \\
\text { inclusions, the gas-liquid ratio is } \\
\text { generally less than } 20 \% \text {, containing } \\
\text { a small amount of } \mathrm{CO}_{2} \text { rich } \\
\text { inclusions, and local fluid inclusions } \\
\text { containing daughter crystals }\end{array}$ & $\begin{array}{l}\text { Basically composed of pure } \mathrm{CO}_{2} \\
\text { inclusions, } \mathrm{CO}_{2} \sim \mathrm{H}_{2} \mathrm{O} \text { three-phase } \\
\text { inclusions, with varying } \mathrm{CO}_{2} / \mathrm{H}_{2} \mathrm{O} \\
\text { ratio }(0-100 \%) \text {, with fluid inclusions } \\
\text { containing daughter crystals locally, } \\
\text { and rich in granulite facies quartz } \\
\text { inclusions in the early stage. }\end{array}$ & $\begin{array}{l}\text { Two-phase inclusions in brine are } \\
\text { dominant, three-phase inclusions of } \\
\mathrm{CO}_{2} \text { are rare, and the gas-liquid } \\
\text { ratio is mostly } 4 \% \text { to } 15 \% \text {. }\end{array}$ \\
\hline $\begin{array}{l}\text { Homogenization temperatures of } \\
\text { the main mineralization periods }\end{array}$ & $\begin{array}{c}130-350{ }^{\circ} \mathrm{C} \text {, the peak value is } \\
180-240^{\circ} \mathrm{C}\end{array}$ & $200-300.0{ }^{\circ} \mathrm{C}$ & $\begin{array}{c}279-406.5^{\circ} \mathrm{C} \text {, the peak value is } \\
320-360^{\circ} \mathrm{C}\end{array}$ & $\begin{array}{c}210-260^{\circ} \mathrm{C} \text { and } 120-170{ }^{\circ} \mathrm{C} \\
\text { (two intervals) }\end{array}$ \\
\hline Pressure & $54-81 \mathrm{MPa}$ & $60.0-72.8 \mathrm{MPa}$ & $\begin{array}{l}\text { Early metallogenic stage } 190-440 \\
\mathrm{MPa} \text {, average } 290 \mathrm{MPa} \text {; main } \\
\text { metallogenic stage } 133.5-340.0 \mathrm{MPa}\end{array}$ & $24-73 \mathrm{MPa}$ \\
\hline Depth & $1.9-3.3 \mathrm{~km}$ & $2.2-2.6 \mathrm{~km}$ & $\begin{array}{c}\text { Early metallogenic stage } 7.2-16.6 \\
\mathrm{~km} \text {, average } 11.0 \mathrm{~km} ; \text { main } \\
\text { metallogenic stage } 5.1-12.9 \mathrm{~km}, \\
\text { average } 9.4 \mathrm{~km}\end{array}$ & $0.90-2.74 \mathrm{~km}$ \\
\hline Salinity w(NaCleqv) \% & $2.89-13.66$, the average of 7.48 & $4.9-11.0$ & $3.70-14.64$, the peak value is $7.2-9.0$ & $6-18$ \\
\hline Eh & Reducing fluid & Reducing fluid & Reducing fluid & Reducing fluid \\
\hline Migration patterns of gold & $\left.\mathrm{Au}(\mathrm{HS})^{2-},[\mathrm{AuAsS}]_{3}\right]^{2-}$ & $\mathrm{Au}(\mathrm{HS})^{2-}$ & $\mathrm{Au}(\mathrm{HS})^{2-}$ & - \\
\hline $\begin{array}{l}\text { The precipitation mechanism } \\
\text { of gold }\end{array}$ & Fluid boiling & - & Fluid immiscibility & - \\
\hline References & {$[22,85-87]$.} & {$[33,91-93]$} & [95-98,100-104,114] & {$[41,48,50,107,111,112]$} \\
\hline
\end{tabular}



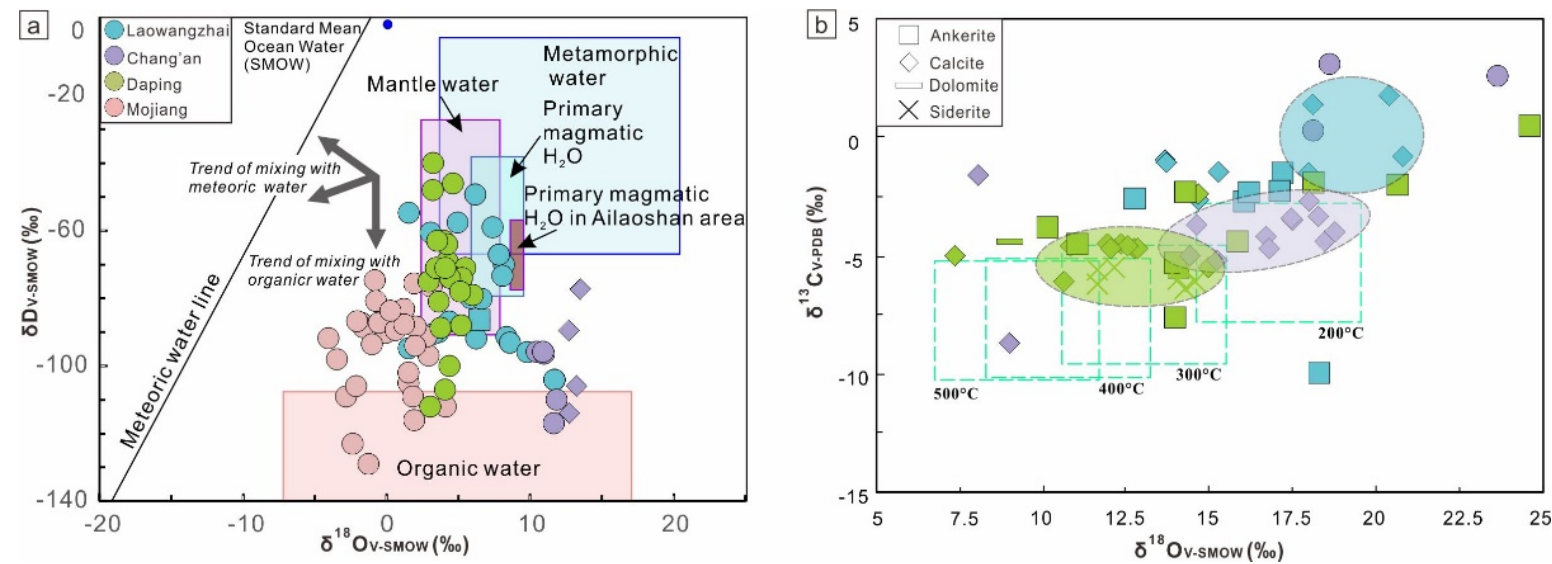

Figure 11. Scatter diagrams of H-O (a) and C-O isotopes (b) in the ore-forming fluid from the Ailaoshan tectonic belt, SW China [121]. The dashed, green box in panel $b$ represents the $\mathrm{C}-\mathrm{O}$ isotopes ranges of calcites expected to precipitate from magmatic water at the indicated temperatures [122]. The $\mathrm{H}-\mathrm{O}$ isotopes of magmatic, metamorphic, and organic fields are from [123]. Note: The different colored ellipses in Figure $11 \mathrm{~b}$ indicate the main scope of " $\delta{ }^{18} \mathrm{O}_{\mathrm{V}-\mathrm{SMOW}}-\delta^{13} \mathrm{C}_{\mathrm{V}-\mathrm{PDB}}$ " in different deposits. And the same color represents the same deposit, and different shapes represent different tested minerals.
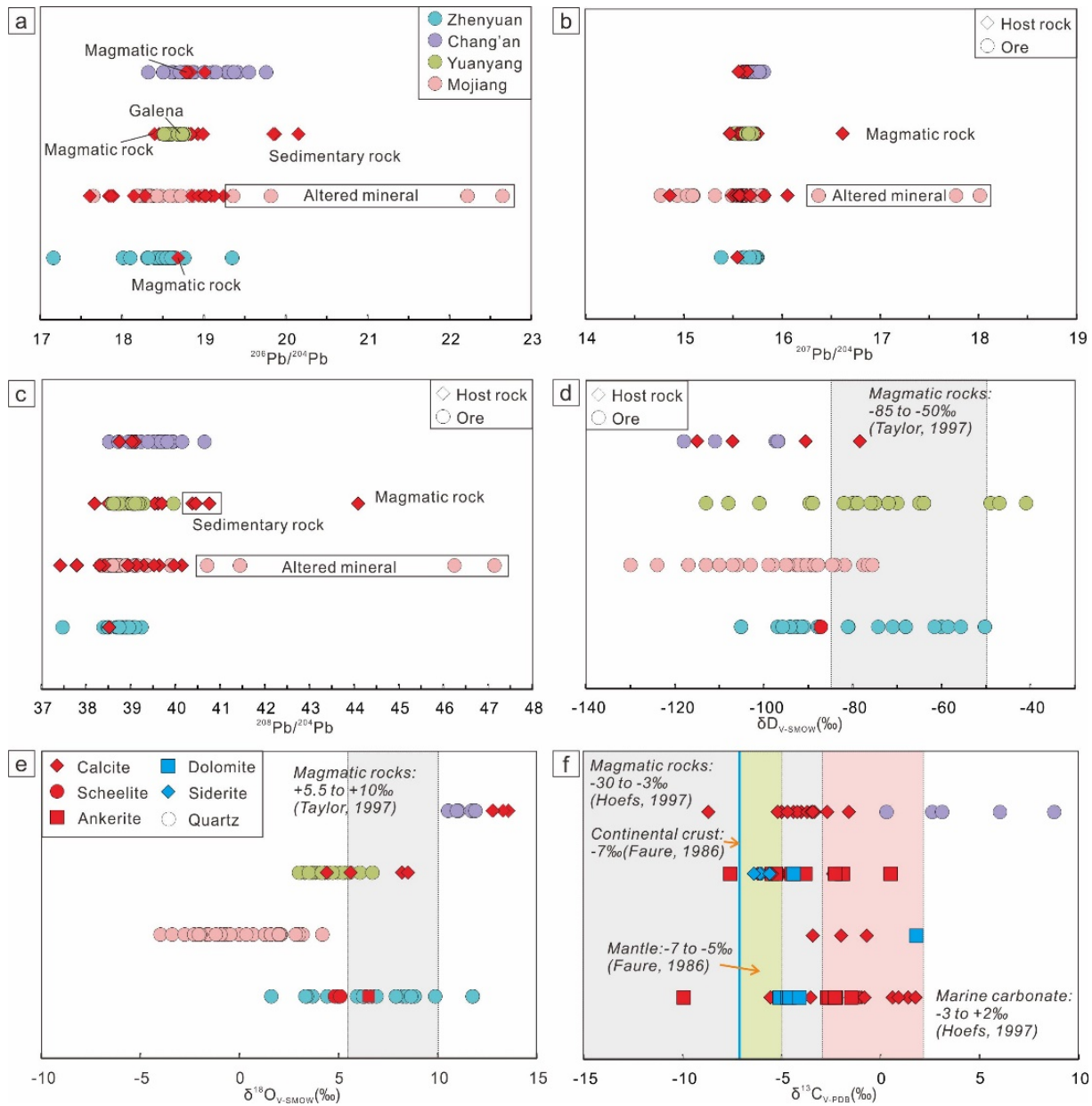

Figure 12. C-H-O-S-Pb isotopes scatter diagram of gold deposits in the Ailaoshan metallogenic belt. (a) ${ }^{206} \mathrm{~Pb} /{ }^{204} \mathrm{~Pb}$. (b) ${ }^{207} \mathrm{~Pb} /{ }^{204} \mathrm{~Pb}$. (c) ${ }^{208} \mathrm{~Pb} /{ }^{204} \mathrm{~Pb}$. (d) D isotopes. (e) O isotopes. (f) $\mathrm{C}$ isotopes. Note: In the figure, the isotopic compositions of different tested minerals in four different deposits are represented from bottom to top: Zhenyuan Laowangzhai, Mojiang Jinchang, Yuanyang Daping and Jinping Chang'an. The same color represents the same deposit, and different shapes represent different tested minerals. 
The H-O isotopes of the Zhenyuan Laowangzhai and Yuanyang Daping gold deposits are similar to those of the magmatic fluid; hence, the ore-forming fluid mostly comes from magmatic water (Taylor, 1974, Figure 11a) [120]. Due to the major changes in the $\delta \mathrm{D}$ values, the four gold deposits are all close to the range of organic water and extend from metamorphic to connate fluid. Deng et al. (2014b) [17] interpreted this as a reflection of isotope exchange as the metamorphic fluid migrated through the sedimentary rocks containing organic matter. The ore-forming fluids in the Ailaoshan metallogenic belt mostly comprise mantle (magmatic water) and metamorphic fluids (metamorphic water), and deposits with shallow mineralization depth are mixed with atmospheric precipitation.

\subsection{Isotopes}

$\mathrm{C}$ isotopes are widely used to trace the source of $\mathrm{CO}_{2}$ in ore-forming fluids and are indicators to determine whether mantle fluids participate in ore-forming processes (Rollinson, 1993; Mao, 2003; Liu et al., 2003; Zhang et al., 2009) [124-127]. By comparing the $\delta^{13} \mathrm{C}_{\mathrm{V}-\mathrm{PDB}}$ carbonate data (calcite, dolomite, iron dolomite, and siderite) in the four main gold deposits in the Ailaoshan metallogenic belt with $\delta^{13} \mathrm{C}$ values of different geological reservoirs (Figures $11 \mathrm{~b}$ and $12 \mathrm{f}$ ), only the $\delta^{13} \mathrm{C}$ of the Daping gold deposit in Yuanyang showed an obvious mantle $(-7 \%$ o to $-5 \%$ ) or continental crust $(-7 \%$ o origin. In the Zhenyuan Laowangzhai and Jinping Chang'an deposits, only a few dolomites and calcite exhibit a mantle or continental crust origin. The $\delta^{13} \mathrm{C}$ values of the Jinping Chang'an, Yuanyang Daping, and Zhenyuan Laowangzhai deposits overlap with the interval of magmatic rocks ( $-30 \%$ to $-3 \%$ ), whereas only one sample in the Mojiang Jinchang deposit overlaps with the interval of magmatic rocks.

Simultaneously, in the four main deposits, the $\delta^{13} \mathrm{C}$ values partially overlap with the variation range of marine carbonate $\delta^{13} \mathrm{C}\left(-3 \%\right.$ o to $2 \%$ ). The $\mathrm{CO}_{2}$ needed in the fluid can be provided by carbonate mineral dissolution and decarbonation, the degassing of the mantle, and crystallization differentiation of magma. During the metallogenic process of the Laowangzhai gold deposit in Zhenyuan, the content of $\mathrm{H}_{2} \mathrm{O}$ in the gas phase of fluid inclusions increases gradually, while the content of $\mathrm{CO}_{2}$ decreases gradually, and the value of $\mathrm{CO}_{2} / \mathrm{H}_{2} \mathrm{O}$ shows a decreasing trend (Zhao, 2014) [22]. This is one of the signs that a fluid is boiling (Phillips and Evans, 2004) [128]. The volatile components escaping from the hydrothermal fluid through boiling are mostly $\mathrm{CO}_{2}, \mathrm{CH}_{4}, \mathrm{H}_{2} \mathrm{~S}$, and $\mathrm{H}_{2} \mathrm{O}$ (Xiong et al., 2015; $\mathrm{Qu}, 2019)[32,46]$. Some isotope fractionation occurs between these gas components and the liquid fluid, and their loss will change the isotope composition in the hydrothermal fluid (Zheng, 2001) [129]. In the process of $\mathrm{CO}_{2}$ degasification, the $\mathrm{CO}_{2}$ released by rocks enriches heavy ${ }^{13} \mathrm{C}$ and ${ }^{18} \mathrm{O}$; therefore, $\delta^{13} \mathrm{C}$ and $\delta^{18} \mathrm{O}$ values of the carbonate minerals formed by residual materials (such as calcite) decrease (Brown et al., 1985; Zheng and Hoefs, 1993) $[130,131]$, increasing the $\delta^{13} \mathrm{C}$ value in the fluid (Zheng et al., 1996) [132]. Based on Zhao's (2014) [22] research on the trace elements and H-O-S isotopes of the golden iron ore in the Zhenyuan Laowangzhai gold deposit, the carbon in the ore-forming fluid is more likely to come from the mantle or continental crust and does not exclude the contamination of marine sedimentary rocks in the shallow part.

The Mojiang Jinchang, Jinping Chang'an, and Yuanyang Daping gold deposits mostly come from the surrounding rocks of the stratum in the mining area; therefore, the carbon is more likely to come from marine carbonate. The Mojiang Jinchang is a Triassic Jinchang Formation siliceous rock, the Jinping Chang'an is a Silurian Kanglang Formation dolomite, and the Yuanyang Daping is a Middle Silurian Kanglang Formation carbonate rock. Among them, the ore-bearing host rock of the Yuanyang Daping gold deposit is Taojiazhai diorite in the north of the mining area and the Middle Silurian Kanglang Formation carbonate rock in the south of the mining area (Du, 2015) [115]. Xiong et al. (2006) [133] found granulite facies graphite inclusions in the scheelite of the Daping gold deposit. They proposed that the carbon in the deposit mostly comes from the original magma formed by the mantle's melting and $\mathrm{CO}_{2}$ mostly comes from the decarbonization of the lower crust, which is affected by weak metasomatism. 


\subsection{Pb Isotopes}

This paper presents the ${ }^{206} \mathrm{~Pb} /{ }^{204} \mathrm{~Pb},{ }^{207} \mathrm{~Pb} /{ }^{204} \mathrm{~Pb}$, and ${ }^{208} \mathrm{~Pb} /{ }^{204} \mathrm{~Pb}$ ratios of different host rocks and ores in the four main gold deposits in the Ailaoshan metallogenic belt. The results showed that the $\mathrm{Pb}$ isotope compositions in the metallogenic zone are similar to some extent, indicating that all gold deposits in the Ailaoshan metallogenic belt might have the same Pb source. Wang et al. (2013) [1] found that the Pb isotope composition of the crust of the Yangtze block can represent the $\mathrm{Pb}$ isotope characteristics of the crust where the Ailaoshan metallogenic belt is located. Therefore, Zhao (2014) [22] collected the Pb isotopic composition of the peraluminous leucogranites produced in the ASRRSZ, representing the characteristics of the upper crust of the Yangtze at the same collision stage, and the $\mathrm{Pb}$ isotopic composition of amphibolite capture bodies in the Eocene potassium-bearing felsic intrusive rocks in western Yunnan to represent the $\mathrm{Pb}$ isotopic characteristics of the middle and lower crust of the Yangtze (Zhao, 2004) [22]. By collecting published $\mathrm{Pb}$ isotope data and putting the data into $\mathrm{Pb}-\mathrm{Pb}$ isotope diagrams (Figures 12 and 13), we can highlight the following details. The $\mathrm{Pb}$ isotopic composition of the four main gold deposits in the Ailaoshan metallogenic belt is slightly different, mostly concentrated between the upper crust and the middle and lower crusts of the Yangtze, showing obvious crust source characteristics. The ${ }^{207} \mathrm{~Pb} /{ }^{204} \mathrm{~Pb}$ and ${ }^{206} \mathrm{~Pb} /{ }^{204} \mathrm{~Pb}$ curves of the Laowangzhai Gold deposit in Zhenyuan shifted to the left, mostly falling in the range of leucogranites in the Ailaoshan metallogenic belt, and partly falling in the amphibolite in the western Yunnan and enriched mantle (EMII) area (Lu et al., 2013, Figure 13a,b) [134].
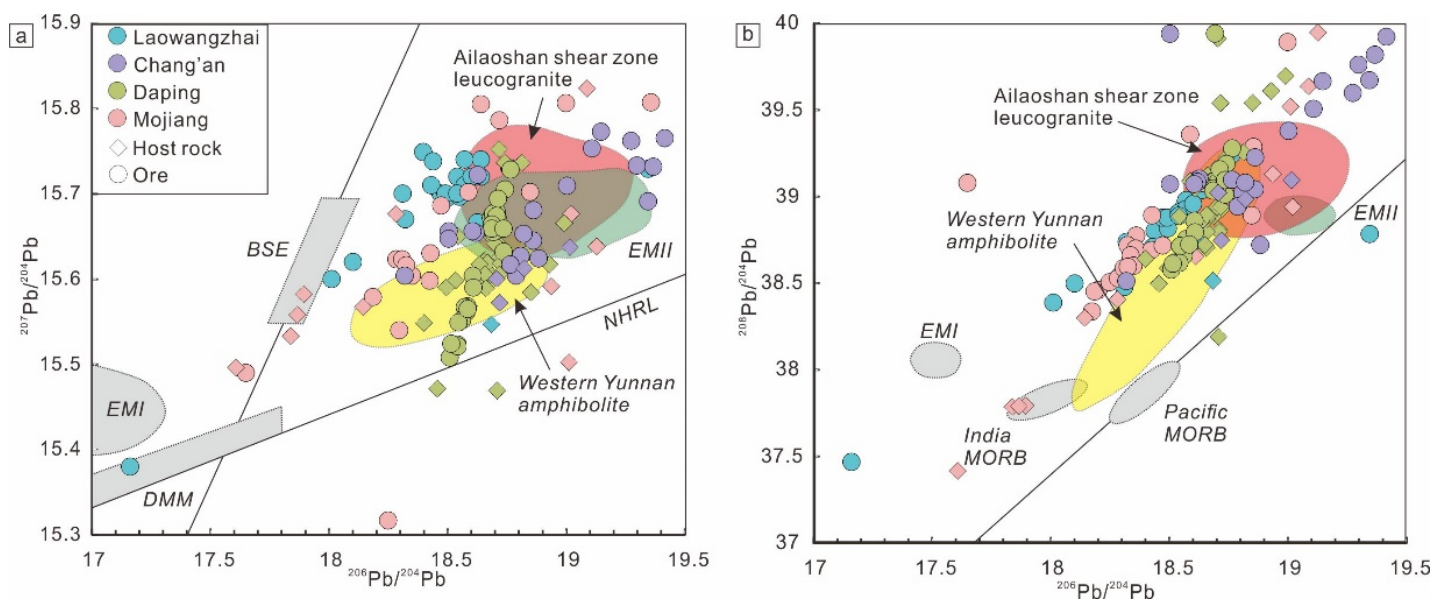

Figure 13. $\mathrm{Pb}$ isotopic composition of sulfide in the Ailaoshan metallogenic belt (modified from [134]). Mantle source reservoirs BSE, MORB, DMM, EM I, and EM II are fromZindler and Hart (1986) [135]. Indian MORB and Pacific MORB are after Guo et al. (2005) [136]. The Northern Hemisphere Reference Line (NHRL) is from Hart (1984) [137]. Data for leucogranite are fromZhang and Schärer (1999) [138] and those for amphibolite xenoliths in the potassic felsic intrusion are fromDeng et al. (1998) [139] and Zhao et al. (2004) [140]. Note: The same color represents the same deposit, and different shapes represent different tested minerals.

Deng et al. (2014b) [17] proposed that the EMII might be caused by the recirculation of upper crust material into the mantle. The $\mathrm{Pb}$ isotopic characteristics show that the $\mathrm{Pb}$ of the Laowangzhai gold deposit in Zhenyuan is mostly derived from the upper crust, accompanied by many mantle-derived materials. The $\mathrm{Pb}$ isotopic characteristics of the Daping gold deposit in Yuanyang overlap with the upper crust and middle and lower crusts. The $\mathrm{Pb}$ isotopes of the Daping gold deposit in Yuanyang are more concentrated in the range of amphiboles in Western Yunnan, and their characteristics are similar to the upper, middle, and lower crusts. Simultaneously, some points are located in the EMII, exhibiting obvious characteristics of crust-mantle mixing. The $\mathrm{Pb}$ isotopic composition of the Jinchang gold-nickel deposit in Mojiang is relatively scattered, and some data are relatively deviated, which might be anomalous $\mathrm{Pb}$. Two of these points fall within the 
block silicate earth range, showing that $\mathrm{Pb}$ mostly comes from the middle and lower crusts, with a small number of other sources involved (Deng et al., 2014b) [17]. The Pb isotopic characteristics of the Chang' an gold deposit in Jinping have similar characteristics to the upper crust, and a small number of points are in the range of the EMII. Therefore, $\mathrm{Pb}$ is more likely to originate from the upper crust with higher maturity, possibly accompanied by a small amount of mantle material. Typically, the $\mathrm{Pb}$ isotopes in the four main gold deposits in the Ailaoshan metallogenic belt have the characteristics of multiple sources. Both the upper crust and the middle and lower crusts are involved, accompanied by the mixing of mantle-derived materials. Some gold deposits have a mixture of stratum $\mathrm{Pb}$ in the shallow part (Zhao, 2014) [22].

The ratio range of ${ }^{207} \mathrm{~Pb} /{ }^{204} \mathrm{~Pb}$ and ${ }^{206} \mathrm{~Pb} /{ }^{204} \mathrm{~Pb}$ of light-colored granite is closer to the upper crust than amphibolite, indicating that light-colored granite is generally formed in the shallower part of the crust compared with amphibolite in western Yunnan. Correspondingly, the source of ore-forming fluids of the Laowangzhai gold deposit in Zhenyuan is shallower than that of the Daping gold deposit in Yuanyang. This is consistent with the result of mineralization depth estimated by measuring the temperatures of fluid inclusions and calculating the captured pressure in the previous article $(1.9-3.3 \mathrm{~km}$ in Zhenyuan; 5.1-12.9 km in Daping).

\subsection{S Isotopes}

This paper summarizes previous research results and analyzes the $S$ isotope characteristics of various deposits in the Ailaoshan metallogenic belt. The study of $\mathrm{S}$ isotopes in ore deposits must analyze the total $\mathrm{S}$ isotope composition of sulfide. In a reducing mineralization system, the $\delta^{34} S$ of sulfide in ore minerals can roughly represent the total S isotope composition of ore-forming hydrothermal fluids (Ohmoto, 1972; Ohmoto and Rye, 1979) [141,142]. Many studies have shown that $S$ in the gold ore-forming fluid in the Ailaoshan area is mostly in the form of $\mathrm{HS}^{-}$and $\mathrm{S}^{2-}$ (Zhao, 2014; Bi et al., 1996; Xie et al., 2001; Xiong et al., 2007; Li, 2012) [22,50,54,92,114]. It shows that the original ore-forming fluid is a reducing fluid, and no sulfate production is found in each gold deposit. Therefore, the $S$ isotope composition $\delta^{34} S$ of the sulfide in each gold deposit can approximately represent the $\mathrm{S}$ isotope composition $\delta^{34} \mathrm{~S}_{\Sigma S}$ of the total sulfur in the ore-forming fluid. In particular, in low-to-medium hydrothermal deposits with low ore-forming temperatures, the total $\mathrm{S}$ isotope composition $\delta^{34} S_{\Sigma \mathrm{S}}$ in the hydrothermal fluid can be represented by $\delta^{34} \mathrm{~S}$ of pyrite (Butler et al., 2004; Zhang et al., 2014; Liu et al., 2013) [41,143,144].

The $\mathrm{S}$ isotope composition analysis shows that the $\delta^{34} \mathrm{~S}$ value of the ore-forming fluid of the Laowangzhai gold deposit in Zhenyuan is distributed in a tower-like manner near the zero value, not significantly different from the $\delta^{34} S$ value of the pyrite in the formation. It shows obvious mantle source characteristics; however, it could be because of the homogenization of deep metamorphic fluids after undergoing metamorphic and alteration processes, indicating that the sulfur source might be the mantle or deep crust and not from the shallow metamorphic rock strata in the mining area. The $\delta^{34} S$ values of various host rocks and ores of the Mojiang Jinchang gold-nickel deposit exhibit a tower-like distribution near $-5 \%$ and are lower than the mantle source $\delta^{34} S$ values. This indicates that the fluid has an extensive material exchange with the surrounding rock, and the homogenization between different sulfur sources is strong. However, the $\delta^{34} S$ value of the altered ultrabasic rock and the closely related nickel ore is low, indicating that the deep-source sulfur in the ore-forming fluid is significantly mixed with the sulfur in the formation, providing more sulfur sources. The $\delta^{34} S$ values of the ore-forming fluids of the Daping gold deposit in Yuanyang are positive, similar to the typical tower-like distribution characteristics of the $S$ isotopes of the Laowangzhai gold deposit in Zhenyuan $\left(+1 \%\right.$ to $+2 \%$ ) , and the value distribution is wide. The $\delta^{34} S$ value is closer to the mantlesourced sulfur and only deviates in the positive direction, indicating that the sulfur in the ore-forming fluid mostly comes from deep sources. The $\delta^{34} \mathrm{~S}$ values of various ores and magmatic rocks and strata in the Jinping Chang'an gold deposit are all concentrated 
around $0 \%$, indicating that different sulfur sources are mixed and uniform. The $\delta^{34} S$ values of some breccia ore and pyrite are low, reflecting that the source of sulfur might be related to the biological sulfur of sedimentation. These characteristics indicate that the sulfur in the ore-forming fluid mostly come from the surrounding rock, and some are mixed with magma sources (Figure 14).

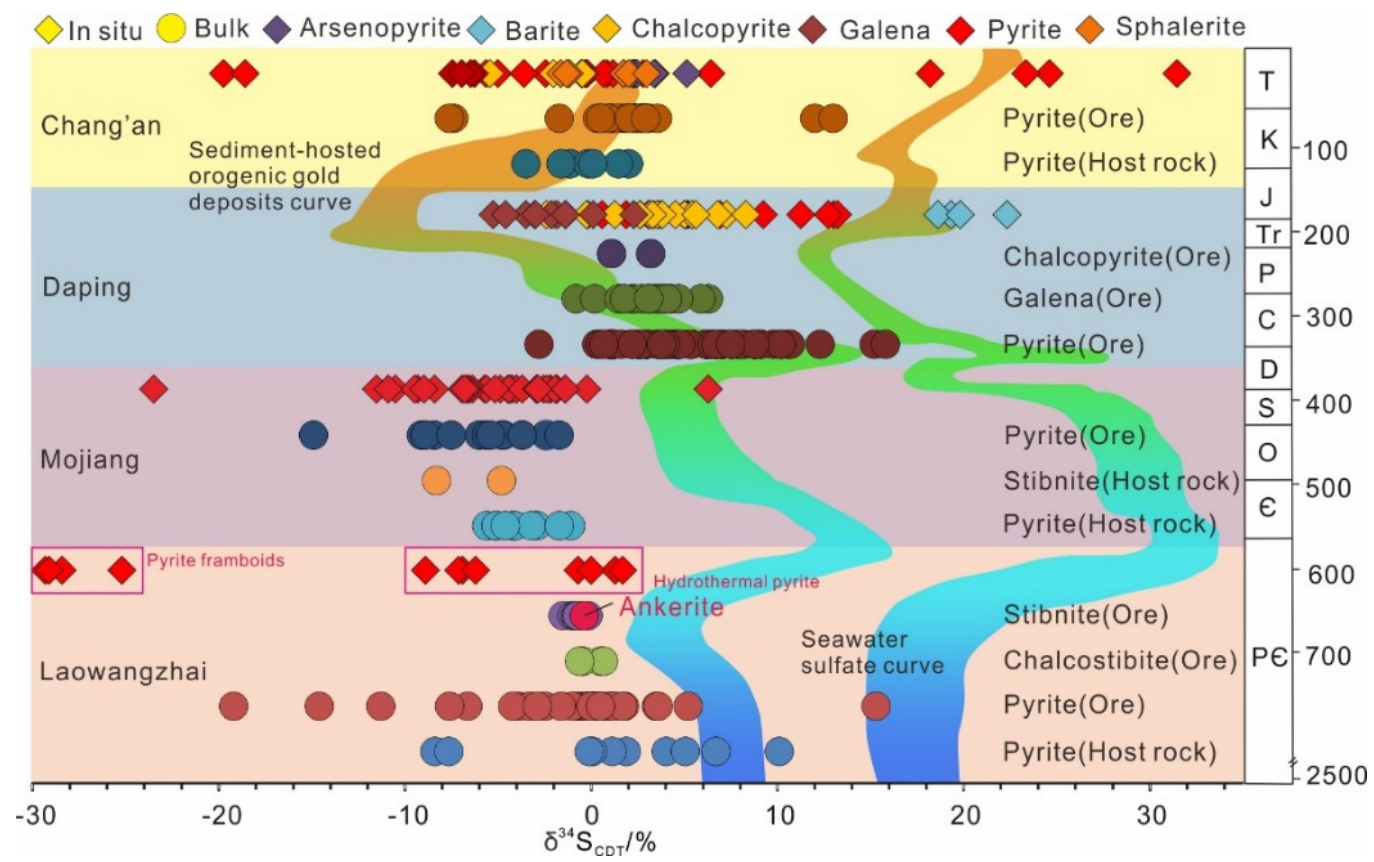

Figure 14. $\mathrm{S}$ isotope composition of sulfide in the Ailaoshan metallogenic belt. The background curve is the $\delta^{34} \mathrm{~S}$ values of seawater carbonate and sedimentary orogenic gold deposits during the geological periods, modified from [145].

\section{Regional Metallogenic Model}

Recently, many scholars considered the crust-mantle interaction as the main metallogenic mechanism of the Ailaoshan metallogenic belt; however, there is still some controversy regarding the source of specific minerals and fluids. From the results of previous studies, the metamorphism of the middle and lower crusts provides mostly metallogenic material and fluid sources in the mineralization zone. The mantle activity might provide the energy required for crust degassing (Powell et al., 1991; Haeussler et al., 1995) [146,147] and the source of some materials and fluids (Rock and Groves, 1988) [148]. A series of Cenozoic porphyry-skarn gold deposits developed in the Ailaoshan metallogenic belt, in which most deposits are related to the Eocene alkali-rich porphyry intrusion (Deng et al., 2015; Hou et al., 2017) $[149,150]$. The amphibolite enclaves in the alkali-rich porphyry body confirmed the existence of the Neoproterozoic lower crust, whereas the high gold and copper content in the amphibolite proved that the metal components originated from the Neoproterozoic lower crust (Hou et al., 2017) [150]. The Neoproterozoic super mantle plume (Figure 15a) and Paleozoic subduction events caused metal enrichment in the young lower crust (Figure 15b), and the partial melting of the Cenozoic lithospheric mantle triggered partial melting of the young lower crust (Figure 15c), forming ore-bearing plutonic rocks (Deng et al., 2014, 2015; Hou et al., 2017) [17,18,149,150].

A close relation exists among the subcontinental lithospheric mantle (SCLM), lower crust, and magmatic-hydrothermal-type gold deposits (Muntean et al., 2011; Hronsky et al., 2012; Griffin et al., 2013; Hou et al., 2017) [150-153]. The original SCLM is deficient in $\mathrm{Au}$ (Rudnick and Gao, 2003) [154] but can be replaced by Au-rich fluids (Wang et al., 2020) [59]. The magma from this enriched SCLM transports Au to the crust. The magma might emplace in the upper part of the crust to form gold deposits or to accumulate at the bottom of the crust to form new metal-rich reservoirs. Furthermore, Au stored in 
the lower crust can be transported to the upper crust during subsequent reactivation. The evolution of the Ailaoshan metallogenic belt is inextricably linked to the tectonic history of the supercontinent of Rodinia, the spread and closure of the Paleo-Tethyan Ocean, subsequent India-Asia continental collision, and postcollisional deformation and exhumation (Wang et al., 2020) [59]. During the Neoproterozoic period, the impact of the mantle plume on the bottom of the South China lithosphere caused heat conduction within the lithosphere, inducing a large proportion of the water-bearing SCLM to undergo partial melting (Li et al., 1999; Li et al., 2002; 2008; Zou et al., 2020) [10-13], forming the lower continental crust and Au-rich SCLM (Figure 15a; Lu et al., 2013; He et al., 2016; Hou et al., 2017; Cai et al., 2014) $[134,150,155,156]$. Both of these regions remobilized and transported gold to the shallow crust (Figure 15c). Hou et al.'s (2017) [150] study on the alkali-rich porphyry internal amphibolite and garnet amphibolite xenolith related to the Beiya gold deposit indicated that xenoliths originated in the lower crust and are the remnants of Neoproterozoic magma. This type of juvenile crust is more enriched in $\mathrm{Cu}, \mathrm{Au}$, and other metallogenic elements than the normal continental crust. The Au in the lower crust is enriched by Eocene magmatic rocks to the surface. Au aggregates and migrates more easily in an environment with a weak melt oxidation state and moderate water content.

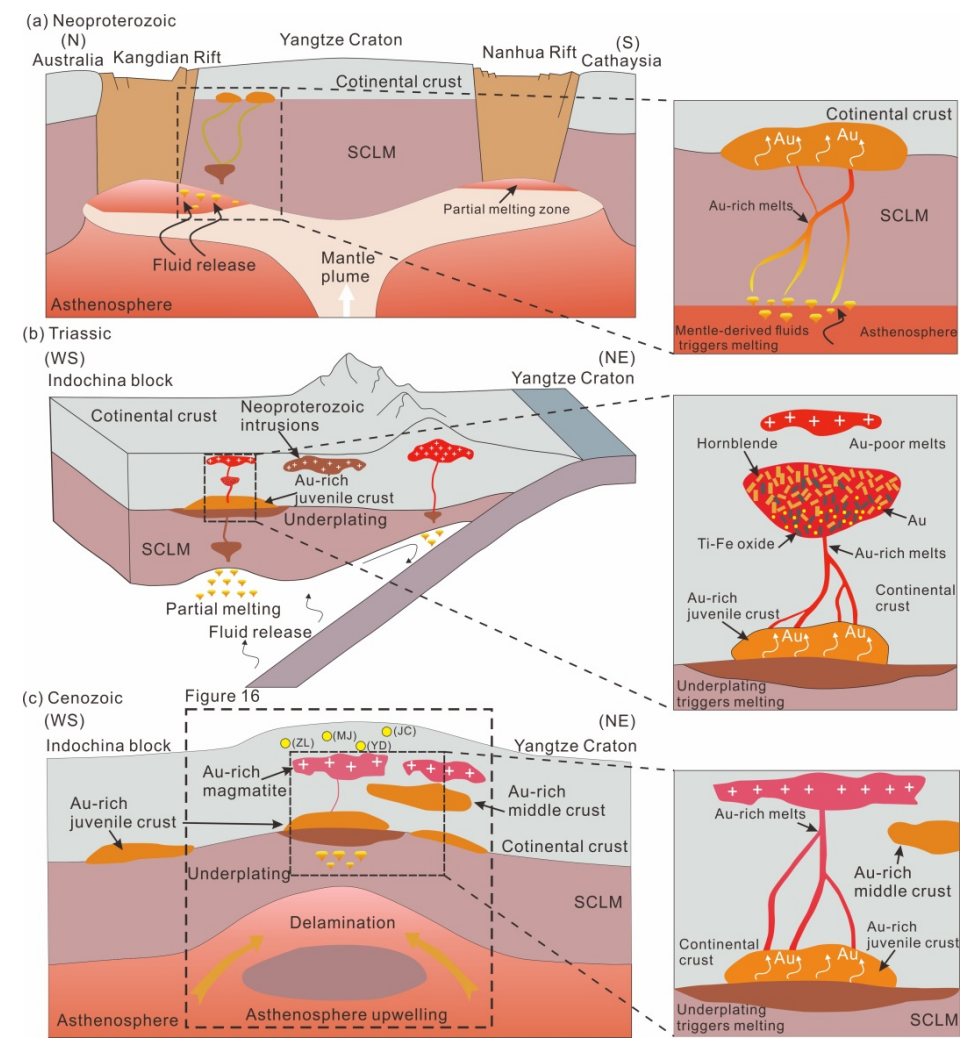

Figure 15. Schematic diagram showing the gold enrichment process in the lithosphere of the western

South China block during the Neoproterozoic, Triassic, and Cenozoic (ZL: Zhenyuan Laowangzhai; MJ: Mojiang Jinchang; YD: Yuanyang Daping; JC: Jinping Chang'an, modified from [59,150,157,158]). (a) and (b). The Neoproterozoic supermantle plume and Paleozoic subduction events caused metal enrichment in the young lower crust. (c). The partial melting of the Cenozoic lithospheric mantle triggered partial melting of the young lower crust, forming ore-bearing plutonic rocks.

However, $\mathrm{Au}$ is precipitated with the crystallization of Ti-Fe oxides in the middle crust (Figure 15b). Consequently, the Au-poor magma emplaces the upper crust and Au-rich middle crust; however, Au might still migrate to the shallow crust to form a localized mineralization zone (Shi et al., 2012) [159]. The postcollisional lithosphere extension after the Cenozoic triggered large-scale magmatic activity (43-32 Ma), accompanied by strong 
shearing and gold mineralization (Figure 15c). The Au-rich lower crustal ore-forming fluids rose through deep faults and formed many gold deposits in the Ailaoshan metallogenic belt. The Au-rich middle crust during the Triassic period is also a probable source of Au in this metallogenic belt (Wang et al., 2020) [59].

The four main gold deposits in the Ailaoshan metallogenic belt are the Laowangzhai in Zhenyuan, the Jinchang in Mojiang, the Daping in Yuanyang, and the Chang'an in Jinping from the west to the east. Metallogenic types include altered rock type, altered rock and quartz vein mixed type, and quartz vein type. Although the mineralization types of the four gold deposits are different, the gold mineralization is consistent in age and all the deposits belong to the early Himalayan orogen. Therefore, they might be under the same tectonic background, enriched by ore-forming fluids of the same periods, and then mineralized. C$\mathrm{H}-\mathrm{O}-\mathrm{S}-\mathrm{Pb}$ isotope data indicate that the main fluid sources of the gold deposits mentioned above are characterized by mixed crust and mantle sources. In summary, the delamination of the lithospheric mantle led to the upwelling of the asthenospheric mantle, and a large number of mantle-derived materials induced deep metamorphism.

The metamorphic hydrothermal fluid moves up and reacts with the surrounding rock and precipitates under suitable metallogenic conditions to form the Ailaoshan gold mineralization zone. Based on the metallogenic characteristics of the gold deposits in the Ailaoshan metallogenic belt, the continuous mineralization mechanism of the crust can be used for reference to establish a regional metallogenic model (Groves, 1993; Groves et al., 1998) $[160,161]$. In the early Himalayas, affected by the continental collision of the IndiaYangtze plate, the Ailaoshan area was squeezed in both directions from the southwest to the northeast, forming a large-scale thrust-nappe tectonic system. Simultaneously, the shear strike-slip fault structure was formed, which significantly shortened and thickened the crust and lithosphere in the Ailaoshan area (Chen et al., 2008) [73]. Strong deformation and metamorphism occurred in the middle and lower crust, accompanied by the activity of mantle-derived magma. The middle and lower crust degassed to form metamorphic $\mathrm{Au}$ and $\mathrm{CO}_{2}$-rich fluids, which might be mixed with some upwelling mantle-derived materials. Subsequently, due to the tectonic system's transformation, many deep shear zones were formed in the Ailaoshan area. The ore-bearing fluid rose along the shear zone, further extracting the surrounding rock-forming minerals, evolving into a high-salinity ore-forming fluid. The fluid is rich in $\mathrm{Au}, \mathrm{Pb}, \mathrm{Cu}$, and other ore-forming elements. When the ore-forming fluid rises to the brittle-ductile transition zone, due to the formation of brittle fractures, the fluid temperatures and pressures drop rapidly, making the $\mathrm{CO}_{2}$-rich ore-bearing fluid immiscible, and fluid boiling allows a large amount of $\mathrm{CO}_{2}$ to escape. This led to the loss of stability of the $\mathrm{Au}(\mathrm{HS})^{2-}$ complex compounds, resulting in a large amount of gold precipitation and the formation of the Daping gold deposit at the tough-brittle structural level.

In the shallow crust, metamorphic fluids, rich in $\mathrm{Au}$, As, and other ore-forming elements produced by metamorphism of the low greenschist facies, are continuously added to the upper crust. The materials from multiple sources in the fluid are fully mixed and uniform: $\mathrm{Au}, \mathrm{As}$, and $\mathrm{S}$ in the ore-forming fluid form $(\mathrm{Au}, \mathrm{As})^{2-},\left(\mathrm{Au}\left[\mathrm{As}, \mathrm{S}_{3}\right]\right)^{2-}$, and other complex compounds, migrating in the hydrothermal fluid. In the early stage of mineralization, $\left(\mathrm{S}_{2}\right)^{2-}$ enters the pyrite lattice in the form of isomorphism. When the fluid migrates to the brittle-ductile structure level, the fluid boils under a reduced pressure and $\mathrm{CO}_{2}$ continues to escape, making the $\left.(\mathrm{AuAsS})_{3}\right)^{2-}$ and $\mathrm{Au}(\mathrm{HS})^{2-}$ complexes in the fluid unstable, triggering the precipitation of gold and forming the Laowangzhai gold deposit. In the ore-forming fluids of the Daping and Laowangzhai gold deposits, mantle-derived fluids (magmatic water) containing $\mathrm{Au}$ and $\mathrm{S}$ are added and atmospheric precipitation might be mixed simultaneously. When the fluid reaches the shallower crust, atmospheric precipitation is continuously added to the ore-forming fluid. The mixed fluid has a strong water-rock reaction with the surrounding rock and extracts $\mathrm{Au}, \mathrm{CO}_{2}$, and $\mathrm{S}$ in the Silurian strata. Simultaneously, as the physical and chemical conditions of the ore-forming fluid change, a large amount of sulfide precipitates in the brittle structural space, reducing the 
activity of the reduced sulfur in the hydrothermal fluid. Consequently, the continuous decomposition of $\mathrm{Au}(\mathrm{HS})^{2-}$ complex compounds precipitated gold and other minerals, forming the Mojiang and Chang' an gold deposits (Figure 16, Zhao, 2014) [22].

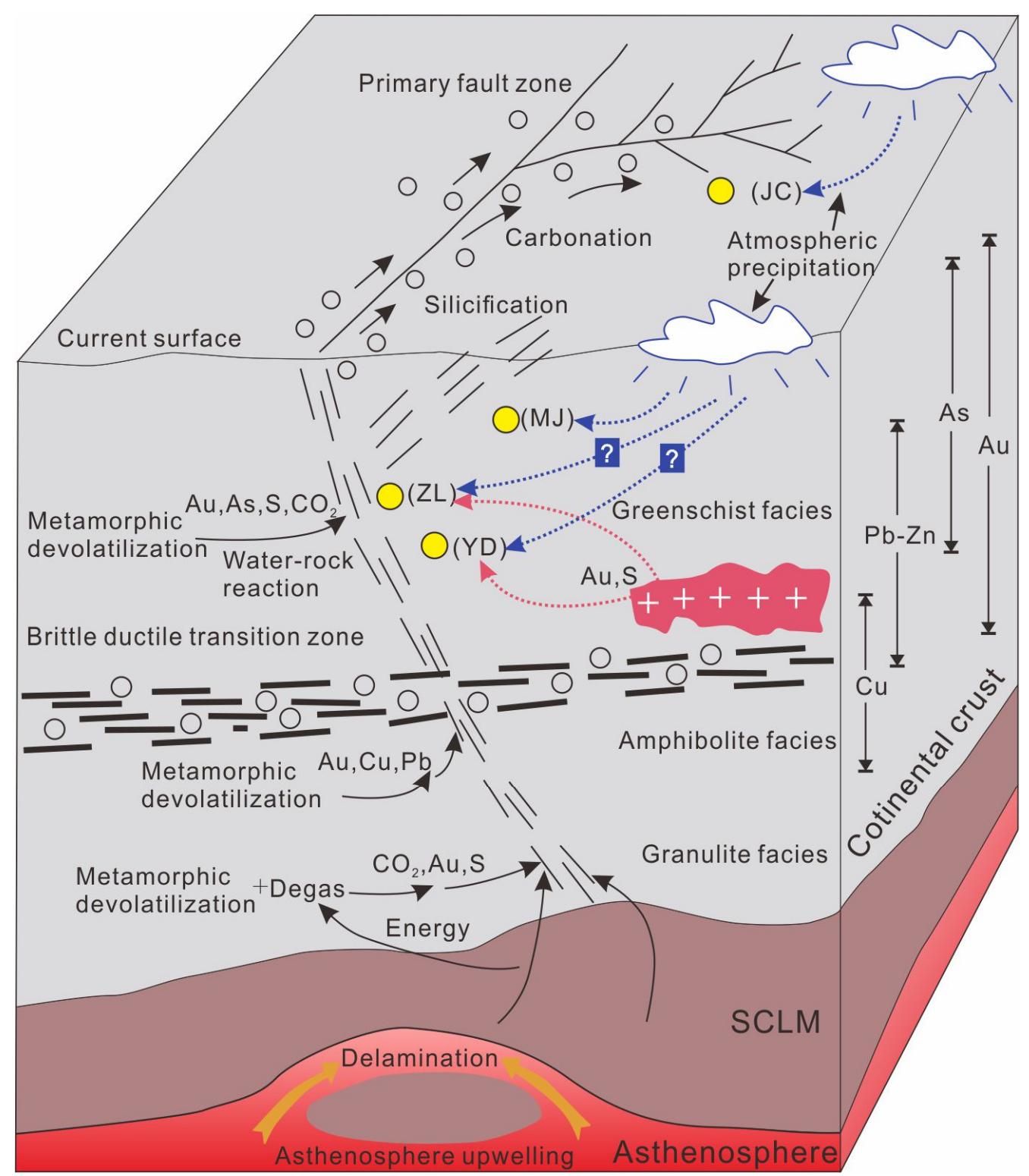

Figure 16. Regional metallogenic model of the Ailaoshan gold belt (ZL: Zhenyuan Laowangzhai; MJ: Mojiang Jinchang; YD: Yuanyang Daping; JC: Jinping Chang'an, modified from [22]).

\section{Conclusions}

(1) The geological characteristic analysis of the four main gold deposits in the Ailaoshan metallogenic belt showed that the area has a strong tectonic-magmatic activity, forming a series of ultrabasic, basic, and intermediate-acid magmatic rocks. The hydrothermal effect is strong, the alteration products are developed, and the spatial zoning of alteration types is not obvious. Gold mineralization is closely related to silicification, argillation, carbonation, and pyrite mineralization.

(2) Based on the existing radiometric age data characteristics in the Ailaoshan metallogenic belt, this paper proposes that the metallogenic-thermal events experienced by the Ailaoshan metallogenic belt can be divided into early (65-60 Ma), major 
(40-30 Ma), and late (30-25 Ma) phases. The gold mineralization mostly occurred between 30 and $50 \mathrm{Ma}$, belonging to the early Himalayan period.

(3) Research on the composition of ore-forming fluid inclusions and the physicochemical properties of ore-forming reveals that the ore-forming fluid in the Ailaoshan metallogenic belt exhibits the characteristics of medium-low temperatures $\left(150-300{ }^{\circ} \mathrm{C}\right)$ and medium-low salinity (an average of around $7 \mathrm{wt} \% \mathrm{NaCl}$ ). The cations in the fluid are mostly $\mathrm{Na}^{+}$and $\mathrm{K}^{+}$, the anions are $\mathrm{Cl}^{-}$and $\mathrm{SO}_{4}{ }^{2-}$, and the gas phase composition mostly comprises $\mathrm{CO}_{2}$; furthermore, the content is between $1 \%$ and $37 \%$, representative of a $\mathrm{H}_{2} \mathrm{O}-\mathrm{NaCl}-\mathrm{CO}_{2}(-\Sigma \mathrm{S})$ system. The fluid is in a neutral, weakly alkaline, and relatively reductive state. Different deposits also exhibit certain differences, mostly reflected in the mineralization temperatures, pressures, depths, and $\mathrm{CO}_{2}$ content. From Yuanyang Daping $\rightarrow$ Zhenyuan Laowangzhai $\rightarrow$ Mojiang Jinchang $\rightarrow$ Jinping Changan, there is a decreasing trend.

(4) The joint study of C-H-O-S-Pb isotopes shows that regional ore-forming fluids mostly comprise mantle (magmatic water) and metamorphic fluids (metamorphic water) and the metamorphic fluids are mostly in the middle and lower crust. Deposits with relatively shallow ore-forming depths might be mixed with meteoric water. The oreforming materials exhibit the characteristics of multiple sources. Both the upper crust and the middle-lower crust are involved, and the mixing of mantle-derived materials also occurs. Some shallow host rocks provide numerous ore-forming materials.

(5) Based on the analysis of the geological characteristics, metallogenic geodynamic background, and genetic mechanism of the gold deposits in the Ailaoshan metallogenic belt, the regional metallogenic model was established by referring to the continuous metallogenic mechanism of the crust combined with the metallogenic characteristics of the Ailaoshan metallogenic belt.

Author Contributions: Conceptualization, D.W. and Y.L.; data curation, Y.L.; writing-original draft preparation, Y.L.; supervision, D.W., Y.L. and C.W.; project administration, Y.S. and M.P.-c., funding acquisition, D.W., Y.L. and Y.S. All authors have read and agreed to the published version of the manuscript.

Funding: This research was funded by [Geology of Mineral Resources in China] grant number [DD20190379], and [Comprehensive investigation and evaluation of a large-scale lithium-beryllium polymetallic resource base in Songpan-Ganzi Lithium Belt] grant number [DD20190173]. And The APC was funded by [DD20190379].

Acknowledgments: The constructive comments and careful revisions from the editor, associate editor, and two reviewers are greatly appreciated. During the writing process of the article, This paper received the help of Cao Huawen, from Chengdu Center, China Geological Survey, Lu Yan, deputy chief engineer; Li Yubin, senior engineer from Tibet Institute of Geological Survey; Liu Hang, from Chengdu Univerisity of Technology; Jiang Xiuwei, from Northwest University, amongothers, and put forward constructive suggestions for the article. This study was financially supported by Geology of Mineral Resources in China, China Geological Survey (DD20190379) and Comprehensive investigation and evaluation of a large-scale lithium-beryllium polymetallic resource base in SongpanGanzi Lithium Belt (DD20190173).

Conflicts of Interest: The authors declare no conflict of interest.

\section{References}

1. Wang, W.C.; Zhang, J.X.; Liu, L.C.; Tang, Y.K.; Liu, X.J. An prospective analysis of ore-searching work in the Bijiashan gold deposit in Dali-Midu area, Yunnan Province. Acta Geosci. Sin. 2013, 34, 108-114.

2. Liu, J.L.; Tang, Y.; Song, Z.J.; Tran, M.D.; Zhai, Y.F.; Wu, W.B.; Chen, W. The Ailaoshan Belt in Western Yunnan: Tectonic framework and tectonic evolution. J. Jilin Univ. 2011, 41, 1285-1303.

3. Metcalfe, I. Tectonic framework and Phanerozoic evolution of Sundaland. Gondwana Res. 2011, 19, 3-21. [CrossRef]

4. Wang, Q.F.; Groves, D.I.; Deng, J.; Li, H.J.; Yang, L.; Dong, C.Y. Evolution of the Miocene Ailaoshan orogenic gold deposits, southeastern Tibet, during a complex tectonic history of lithosphere-crust interaction. Miner. Depos. 2020, 55, 1085-1104. [CrossRef] 
5. Wang, Q.F.; Zhao, H.S.; Groves, D.I.; Deng, J.; Zhang, Q.W.; Xue, S.C. The Jurassic Danba hypozonal orogenic gold deposit, western China: Indirect derivation from fertile mantle lithosphere metasomatized during Neoproterozoic subduction. Miner. Depos. 2020, 55, 309-324. [CrossRef]

6. Schärer, U.; Tapponnier, P.; Lacassin, R.; Leloup, P.H.; Zhong, D.; Ji, S. Intraplate tectonics in Asia: A precise age for large-scale Miocene movement along the Ailao Shan-Red River shear zone, China. Earth Planet. Sci. Lett. 1990, 97, 65-77. [CrossRef]

7. Leloup, P.H.; Lacassin, R.; Tapponnier, P.; Schrer, U.; Zhong, D.L.; Liu, X.H.; Zhang, L.S.; Ji, S.C.; Trinh, P.T. The Ailao Shan-Red River shear zone (Yunnan, China), Tertiary transform boundary of Indochina. Tectonophysics 1995, 251, 3-84. [CrossRef]

8. Hou, Z.Q.; Cook, N.J. Metallogenesis of the Tibetan collisional orogen: A review and introduction to the special issue. Ore Geol. Rev. 2009, 36, 2-242. [CrossRef]

9. Hou, Z.Q.; Duan, L.F.; Lu, Y.J.; Zheng, Y.C.; Zhu, D.C.; Yang, Z.M.; Yang, Z.S.; Wang, B.D.; Pei, Y.R.; Zhao, Z.D.; et al. Lithospheric architecture of the Lhasa terrane and its control on ore deposits in the Himalayan-Tibetan orogen. Econ. Geol. 2015, 110, 1541-1575. [CrossRef]

10. Li, Z.X.; Li, X.H.; Kinny, P.D.; Wang, J. The breakup of Rodinia: Did it start with a mantle plume beneath South China? Earth Planet. Sci. Lett. 1999, 173, 171-181. [CrossRef]

11. Li, X.H.; Li, W.X.; Li, Z.X.; Ying, L. 850-790 Ma bimodal volcanic and intrusive rocks in northern Zhejiang, South China: A major episode of continental rift magmatism during the breakup of Rodinia. Lithos 2008, 102, 341-357. [CrossRef]

12. Li, X.H.; Li, Z.X.; Zhou, H.; Liu, Y.; Kinny, P.D. U-Pb zircon geochronology, geochemistry and Nd isotopic study of Neoproterozoic bimodal volcanic rocks in the Kangdian Rift of South China: Implications for the initial rifting of Rodinia. Precambrian Res. 2002, 113, 135-154. [CrossRef]

13. Zou, H.; Bagas, L.; Li, X.Y.; Liu, H.; Jiang, X.W.; Li, Y. Origin and evolution of the Neoproterozoic Dengganping Granitic Complex in the western margin of the Yangtze Block, SW China: Implications for breakup of Rodina Supercontinent. Lithos 2020, 370-371, 105602. [CrossRef]

14. Liu, H.C.; Wang, Y.J.; Fan, W.M.; Zi, J.W.; Cai, Y.F.; Yang, G.L. Petrogenesis and tectonic implications of Late-Triassic high $\varepsilon \mathrm{Nd}(\mathrm{t})-\varepsilon H f(t)$ granites in the Ailaoshan tectonic zone (SW China). Sci. China Earth Sci. 2014, 57, 2181-2194. [CrossRef]

15. Liu, H.C.; Wang, Y.J.; Cawood, P.A.; Fan, W.M.; Cai, Y.F.; Xing, X.W. Record of Tethyan ocean closure and Indosinian collision along the Ailaoshan suture zone (SW China). Gondwana Res. 2015, 27, 1292-1306. [CrossRef]

16. Yang, L.; Wang, Q.F.; Wang, Y.N.; Li, G.J. Proto- to Paleo-Tethyan evolution of the eastern margin of Simao block. Gondwana Res. 2018, 62, 61-74. [CrossRef]

17. Deng, J.; Wang, Q.F.; Li, G.J.; Santosh, M. Cenozoic tectono-magmatic and metallogenic processes in the Sanjiang region, southwestern China. Earth-Sci. Rev. 2014, 138, 268-299. [CrossRef]

18. Deng, J.; Wang, Q.F.; Li, G.J.; Li, C.S.; Wang, C.M. Tethys tectonic evolution and its bearing on the distribution of important mineral deposits in the Sanjiang region, SW China. Gondwana Res. 2014, 26, 419-437. [CrossRef]

19. Deng, J.; Wang, Q.F.; Li, G.J. Tectonic evolution, superimposed orogeny, and composite metallogenic system in China. Gondwana Res. 2017, 50, 216-266. [CrossRef]

20. Li, P. Ore-Forming Fluids Evolution of the Laowangzhai Gold Deposit, Western Yunnan. Master Thesis, China University of Geosciences, Beijing, China, 2014. (In Chinese with English abstract).

21. Sun, X.M.; Zhang, Y.; Xiong, D.X.; Sun, W.D.; Shi, G.Y.; Zhai, W.; Wang, S.W. Crust and mantle contributions to gold-forming process at the Daping deposit, Ailaoshan gold belt, Yunnan, China. Ore Geol. Rev. 2009, 36, 235-249. [CrossRef]

22. Zhao, K. Geochemistry of Ore-Forming Processes in the Ailaoshan Orogenic Gold Belt, Yunnan. Ph.D. Thesis, China University of Geosciences, Beijing, China, 2014. (In Chinese with English abstract).

23. Yang, L.Q.; Deng, J.; Zhao, K.; Liu, J.T. Tectono-thermochronology and gold mineralization events of orogenic gold deposits in Ailaoshan orogenic belt, Southwest China: Geochronological constraints. Acta Petrol. Sin. 2011, 27, 2519-2532, (In Chinese with English abstract).

24. Deng, J.; Hou, Z.Q.; Mo, X.X.; Yang, L.Q.; Wang, Q.F.; Wang, C.M. Superimposed orogenesis and metallogenesis in Sanjiang Tethys. Miner. Depos. 2010, 29, 37-42, (In Chinese with English abstract).

25. Deng, J.; Yang, L.Q.; Ge, L.S.; Yuan, S.S.; Wang, Q.F.; Zhang, J.; Gong, Q.J.; Wang, C.M. Character and post-ore changes, modifications and preservation of Cenozoic alkali-rich porphyry gold metallogenic system in western Yunnan, China. Acta Petrol. Sin. 2010, 26, 1633-1645, (In Chinese with English abstract).

26. Tapponnier, P.; Lacassin, R.; Leloup, P.H.; Schärer, U.; Zhong, D.L.; Wu, H.W.; Liu, X.H.; Ji, S.C.; Zhang, L.S.; Zhong, J.Y. The Ailao Shan/Red River metamorphic belt: Tertiary left-lateral shear between Indochina and South China. Nature 1990, 343, 431-437. [CrossRef]

27. Goldfarb, R.J.; Groves, D.I.; Gardoll, S. Orogenic gold and geologic time: A global synthesis. Ore Geol. Rev. 2001, 18, 1-75. [CrossRef]

28. Faure, M.; Lin, W.; Yang, C.; Lepvrier, C. Triassic tectonics of the Ailaoshan Belt (SW China): Early Triassic collision between the South China and Indochina blocks, and Middle Triassic intracontinental shearing. Tectonophysics 2016, 683, 27-42. [CrossRef]

29. Bozzo, A.T.; Chen, H.-S.; Kass, J.R.; Barduhn, A.J. The properties of the hydrates of chlorine and carbon dioxide. Desalination 1975, 16, 303-320. [CrossRef]

30. Lu, H.Z.; Fan, H.R.; Ni, P. The Fluild Inclusion; Science Publishing House: Beijing, China, 2004; pp. 1-488. (In Chinese) 
31. Fang, W.X.; Hu, R.Z.; Xie, G.Q.; Su, W.C.; Qi, L. Diagenetic-metallogenic ages of pyritic cherts and their implications in Mojiang nickel-gold deposit in Yunnan Province. Chin. Sci. Bull. 2001, 46, 1823-1827. [CrossRef]

32. Xiong, Y.Q.; Yang, L.Q.; Shao, Y.J.; Zhao, K.; Li, P.; Lu, Y.G.; Du, D.Y. Metallogenic process in Jinchang gold-nickel deposit, Mojiang County, SW Yunnan, China: Constraints from occurrence of gold and nickel. Acta Petrol. Sin. 2015, 31, 3309-3330, (In Chinese with English abstract).

33. Ying, H.L.; Wang, D.H.; Liu, H.L. Geology and formation time of nickel mineralization in Jinchang nickel-gold deposit, Mojiang, Yunnan. Miner. Depos. 2005, 24, 44-51, (In Chinese with English abstract).

34. BGMRYP (Bureau of Geology and Mineral Resources of Yunnan Province). Exploration Report of the Donggualin Ore Body Cluster, Zhenyuan Deposit; BGMRYP: Yunnan, China, 1993. (In Chinese)

35. BGMRYP (Bureau of Geology and Mineral Resources of Yunnan Province). Exploration Report of the Laowangzhai Orebody Cluster, Zhenyuan Deposit; BGMRYP: Yunnan, China, 1993. (In Chinese)

36. Yang, L.Q.; Liu, J.T.; Zhang, C.; Wang, Q.F.; Ge, L.S.; Wang, Z.L.; Zhang, J.; Gong, Q.J. Superimposed orogenesis and metallogenesis: An example from the orogenic gold deposits in Ailaoshan gold belt, Southwest China. Acta Petrol. Sin. 2010, 26, 1723-1739, (In Chinese with English abstract).

37. Deng, J.; Wang, Q.F.; Li, G.J.; Zhao, Y. Structural control and genesis of the Oligocene Zhenyuan orogenic gold deposit, SW China. Ore Geol. Rev. 2015, 65, 42-54. [CrossRef]

38. Huang, Z.L.; Liu, C.Q.; Yang, H.L.; Xu, C.; Han, R.S.; Xiao, H.Y.; Zhang, B.; Li, W.B. Geochemistry of lamprophyres in Laowangzhai gold deposits, Yunnan Province, China: Implication for its characteristics of source region. Geochem. J. 2002, 39, 91-112.

39. Yang, L. Tectonic Evolution and Gold Mineralization in the Southwest Tethyan Ailaoshan Belt. Ph.D. Thesis, China University of Geosciences, Beijing, China, 2018. (In Chinese with English abstract).

40. Chen, Y.H.; Yao, S.Z.; Pan, Y.M. Geochemistry of lamprophyres at the Daping gold deposit, Yunnan province, China: Constraints on the timing of gold mineralization and evidence for mantle convection in the eastern Tibetan Plateau. J. Asian Earth Sci. 2014, 93, 129-145. [CrossRef]

41. Zhang, J.; Deng, J.; Chen, H.Y.; Yang, L.Q.; Cooke, D.; Danyushevsky, L. LA-ICP-MS trace element analysis of pyrite from the Chang'an gold deposit, Sanjiang region, China: Implication for ore-forming process. Gondwana Res. 2014, 26, 557-575. [CrossRef]

42. Zhang, Y.Y.; Zhang, D.; Wu, G.G.; Di, Y.J.; Li, X.J.; Bu, X.C.; Liu, J. Origin of the Daping gold deposit in the Ailaoshan metallogenic belt, SW China: Insights from geology, isotope geochemistry and geochronology. Ore Geol. Rev. 2018, 96, 1-12. [CrossRef]

43. Liang, Y.H.; Sun, X.M.; Shi, G.Y.; Hu, B.M.; Zhou, F.; Wei, H.X.; Mo, R.W. Ore-forming fluid geochemistry and genesis of Laowangzhai large scale orogenic gold deposit in Ailaoshan gold belt, Yunnan Province, China. Acta Petrol. Sin. 2011, 27, 2533-2540, (In Chinese with English abstract).

44. Li, Y. Discussion on metallogenic material source of Mojiang gold deposit. Yunnan Geol. 1992, 11, 130-143, (In Chinese with English abstract).

45. Fan, W.M.; Wang, Y.J.; Zhang, A.M.; Zhang, F.F.; Zhang, Y.Z. Permian arc-back-arc basin development along the Ailaoshan tectonic zone: Geochemical, isotopic and geochronological evidence from the Mojiang volcanic rocks, Southwest China. Lithos 2010, 119, 553-568. [CrossRef]

46. Qu, J.F. Evolution of Ore-Forming Fluids and Metallogenesis of the Jinchang Au Deposit in Mojiang County, Yunnan Province. Master Thesis, China University of Geosciences, Beijing, China, 2019. (In Chinese with English abstract).

47. Chen, Y.H. Metallogenic Conditions and Gold Enrichment Regularity in the Daping Gold Deposit, Yunnan Province, P.R. China. Ph.D. Thesis, China University of Geosciences, Beijing, China, 2015. (In Chinese with English abstract).

48. Yang, L.; Wang, Q.F.; Large, R.R.; Mukherjee, I.; Deng, J.; Li, H.J.; Yu, H.Z.; Wang, X. Fluid source and metal precipitation mechanism of sediment-hosted Chang'an orogenic gold deposit, SW China: Constraints from sulfide texture, trace element, $\mathrm{S}, \mathrm{Pb}$, and He-Ar isotopes and calcite C-O isotopes. Am. Mineral. 2021, 106, 410-429. [CrossRef]

49. Li, N.; Chen, Y.J.; Santosh, M.; Pirajno, F. Compositional polarity of Triassic granitoids in the Qinling Orogen, China: Implication for termination of the north-ernmost paleo-Tethys. Gondwana Res. 2013, 27, 244-257. [CrossRef]

50. Li, S.H. The Metallogenesis of the Chang'an Gold Deposit in Southern Ailaoshan Metallogenic Belt. Master Thesis, China University of Geosciences, Beijing, China, 2012. (In Chinese with English abstract).

51. Wang, D.H.; Qu, W.J.; Li, Z.W.; Yin, H.L.; Chen, Y.C. Mineralization episode of porphyry copper deposits in the Jinshajiang-Red River mineralization belt: Re-Os dating. Ser. D Earth Sci. 2005, 48, 192-198. [CrossRef]

52. Wang, D.H.; Mao, J.W.; Yan, S.H.; Yang, J.M.; Xu, J.; Chen, Y.H.; Xue, C.J. Episodes of Cenozoic Gold Mineralization on the Eastern Margin of the Qinghai-Tibet Plateau: ${ }^{40} \mathrm{Ar} /{ }^{39} \mathrm{Ar}$ Dating and Implication for Geodynamic Events. Acta Geol. Sin. 2005, 79, 233-253.

53. Hu, Y.Z.; Tang, S.C.; Wang, H.P. Geology of Gold Deposits in Ailaoshan; Geological Publishing House: Beijing, China, 1995; pp. 1-273. (In Chinese)

54. Bi, X.W.; Hu, R.Z.; He, M.Y. Discussion on metallogenic age and metallogenic mechanism of Ailaoshan gold belt. Geochemistry 1996, 1, 94-97. (In Chinese)

55. Wang, J.H.; Yin, A.; Harrison, T.M.; Grove, M.; Zhang, G.H.; Xie, Y.Q. A tectonic mode for Cenozoic igneous activities in the eastern Indo/Asian collision zone. Earth Planet. Sci. Lett. 2001, 188, 123-133. [CrossRef]

56. Tang, S.C.; Li, J.D.; He, S.X. On metallogenic regularity of gold metallogenic belts in northern section of Ailao Mountain. Yunnan Geol. 1991, 10, 44-70, (In Chinese with English abstract). 
57. Li, G.X. Structural analysis of the Laowangzhai gold orefield Ailaoshan Mountain. In Contribution to the Geology of the QinghaiXizang (Tibet) Plateau; China Geological Press: Beijing, China, 1990; Volume 20, pp. 187-197, (In Chinese with English abstract).

58. The Third Geological Team of Yunnan Bureau of Geology and Mineral Resources. 1993; unpublished.

59. Wang, Y.; Zhang, H.R.; Zhang, H.C.; Chai, P.; Hou, Z.Q. Gold in the lithosphere of the western South China Block, SW China: Insights from quartz porphyries from the giant Zhenyuan gold deposit. Ore Geol. Rev. 2020, 119, 103312. [CrossRef]

60. Tan, X.C.; You, W.D. Regionally metallogenic geological setting of major non-ferrous metal deposits in western Yunnan. Yunnan Geol. 1991, 10, 11-43, (In Chinese with English abstract).

61. Yang, Z.Y.; Hu, G.Y.; Zhao, X.Y. New ${ }^{40} \mathrm{Ar} /{ }^{39} \mathrm{Ar}$ Data of Gold Mineralization in the Ailaoshan Gold Belt, Yunnan Province, China. Acta Geol. Sin. 2020, 94, 210-211. [CrossRef]

62. Sun, X.M.; Xiong, D.X.; Shi, G.Y.; Wang, S.W.; Zhai, W. ${ }^{40} \mathrm{Ar} /{ }^{39} \mathrm{Ar}$ Dating of Gold Deposit Hosted in the Daping Ductile Shear Zone in the Ailaoshan Gold Belt, Yunnan Province, China. Acta Geol. Sin. 2007, 81, 88-93, (In Chinese with English abstract).

63. Zhang, Y.; Sun, X.M.; Shi, G.Y.; Xiong, D.X.; Wei, Z. SHRIMP U-Pb dating of zircons from diorite batholith hosting Daping gold deposit in ailaoshan gold belt, Yunan province, China. Acta Petrol. Sin. 2011, 27, 2600-2608, (In Chinese with English abstract).

64. Luo, Y.N.; Yu, R.L. Orogenic Evolution and Metallogenic Time-Space Distribution in Jinshajiang-Lancangjiang-Nujiang Region, Southwest China. Acta Geosci. Sin. 2002, 5, 417-422, (In Chinese with English abstract).

65. Fu, D.M.; Xu, M.J. Geology of Xiacun AG-Polymetallic ore deposit in West Sichuan, China and Correlation with Kuroko deposits. Acta Geol. Sichuan 1996, 16, 67-72, (In Chinese with English abstract).

66. Wang, Y. The Geological Characteristics and Metallogenetic Model of the Chang'an Gold Deposit in the Jinping County, Yunnan Province. Ph.D. Thesis, Chinese Academy of Geological Sciences, Beijing, China, 2008. (In Chinese with English abstract).

67. Xu, L.L.; Bi, X.W.; Hu, R.Z.; Zhang, X.C.; Su, W.C.; Qu, W.J.; Hu, Z.C.; Tang, Y.Y. Relationships between porphyry Cu-Mo mineralization in the Jinshajiang-Red River metallogenic belt and tectonic activity: Constraints from zircon U-Pb and molybdenite Re-Os geochronology. Ore Geol. Rev. 2012, 48, 460-473. [CrossRef]

68. Zhang, J.; Wang, H.; Li, S.H.; Li, T.J. Paleogene magmatism and gold metallogeny of the Jinping terrane in the Ailaoshan ore belt, Sanjiang Tethyan Orogen (SW China): Geology, deposit type and tectonic setting. Ore Geol. Rev. 2017, 91, 620-637. [CrossRef]

69. Liang, H.Y.; Campbell, I.H.; Allen, C.M.; Sun, W.D.; Yu, H.X.; Xie, Y.W.; Zhang, Y.Q. The age of the potassic alkaline igneous rocks along the Ailao Shan-Red River shear zone: Implications for the onset age of left-lateral shearing. J. Geol. 2007, 115, 231-242. [CrossRef]

70. Zhang, Y.Q.; Xie, Y.W. Geochronology of Ailaoshan-Jinshajiang alkali rich intrusive rocks and their Sr and Nd isotopic characteristics. Ser. D Earth Sci. 1997, 40, 524-530. [CrossRef]

71. Huang, B.; Liang, H.Y.; Mo, J.H.; Xie, Y.W. Zircon LA-ICP-MS U-Pb age of the Jinping-Tongchang porphyry associated with Cu-Mo mineralization and its geological implication. Geotecton. Metallog. 2009, 33, 598-602, (In Chinese with English abstract).

72. Deng, J.; Yang, L.Q.; Wang, C.M. Research advances of superimposed orogenesis and metallogenesis in the Sanjiang Tethys. Acta Petrol. Sin. 2011, 29, 37-42, (In Chinese with English abstract).

73. Chen, J.P.; Tang, J.X.; Chen, Y.; Li, B.H.; Shang, B.C. Geological Characteristics and Metallogenic Model of the Narigongma Copper-Molybdenum Deposit in the North Part of the Sanjiang Region, Southwest China. Geoscience 2008, 22, 9-17, (In Chinese with English abstract).

74. Funahara, S.; Nishiwaki, N.; Murata, F.; Otofuji, Y.I.; Wang, Y.Z. Clockwise rotation of the Red River fault inferred from paleomagnetic study of Cretaceous rocks in the Shan-Thai-Malay block of western Yunnan, China. Earth Planet. Sci. Lett. 1993, 117, 29-42. [CrossRef]

75. Funahara, S.; Nishiwaki, N.; Miki, M.; Murata, F.; Wang, Y.Z. Paleomagnetic study of Cretaceous rocks from the Yangtze block, central Yunnan, China: Implications for the India-Asia collision. Earth Planet. Sci. Lett. 1992, 113, 77-91. [CrossRef]

76. Huang, K.; Opdyke, N.D. Paleomagnetic results from Cretaceous and Jurassic rocks of South and Southwest Yunnan: Evidence for large clockwise rotations in the Indochina and Shan-Thai-Malay terranes. Earth Planet. Sci. Lett. 1993, 117, 507-524. [CrossRef]

77. Chen, H.H.; Dobson, J.; Heller, F.; Hao, J. Paleomagnetic evidence for clockwise rotation of the Simao region since the Cretaceous: A consequence of India-Asia collision. Earth Planet. Sci. Lett. 1995, 134, $203-217$.

78. Sato, K.; Liu, Y.Y.; Zhu, Z.C.; Yang, Z.Y.; Otofuji, Y. Paleomagnetic study of Middle Cretaceous rocks from Yunlong western Yunnan, China: Evidence of southward displacement of Indochina. Earth Planet. Sci. Lett. 1999, 165, 1-15. [CrossRef]

79. Li, D.M.; Cao, Z.M.; Qin, G.X.; He, S.X.; Li, B.H.; Wen, C.Q.; Xu, Z.M. Gold Deposit in Ailaoshan Ophiolite Melange Belt; Geological Publishing House: Beijing, China, 1998; pp. 1-137. (In Chinese)

80. Deng, B.P.; Liu, X.F.; Lu, Q.X. Sr-Nd-Pb Isotopic Compositions and Their Geological Implications of the Laowangzhai Gold Deposit. Sci. Technol. Eng. 2013, 13, 7947-7952, 7965, (In Chinese with English abstract).

81. Deng, B.P.; Liu, X.F.; Lu, Q.X.; Zhao, F.F. Isotope Compositions and their Geological Implications of Laowangzhai Gold Deposit. Met. Mine 2013, 445, 109-112, 117, (In Chinese with English abstract).

82. Deng, B.P.; Liu, X.F.; Zhang, M.; Lu, Q.X.; Zhao, F.F.; Li, C.H.; Chu, Y.T.; Xu, Y.Y.; Tian, X.M.; Liu, H. The petrographical and isotope geochemical tracers for deep ore-forming fluids from the Laowangzhai gold depoist in the northern part of the Ailao Mountains. Chin. J. Geochem. 2013, 32, 281-294. [CrossRef]

83. Mo, X.X.; Zhao, Z.D.; Yu, X.H. Cenozoic Collision-Post Collisional Igneous Rocks in the Tibetan Plateau; Geological Publishing House: Beijing, China, 2009; pp. 1-396. (In Chinese) 
84. Huang, Z.L.; Wang, L.K.; Zhu, C.M. Elements activity during lamprophyre alteration in Zhenyuan gold deposit, Yunnan. Geotecton. Metallog. 1996, 20, 245-254.

85. Huang, Z.L.; Xiao, H.Y.; Zhu, D.; Wang, L.K. The law of element activities in the processes of mineralization for lamprophyres in Zhenyuan gold orefield, Yunnan province. Acta Mineral. Sin. 1998, 3, 337-343, (In Chinese with English abstract).

86. Zhang, J.W.; Wu, J.; Li, C.S.; Su, X.J.; Wang, J.P. Geological features and genesis of Laowangzhai gold deposit in Zhenyuan county, Yunnan. Gold 2010, 31, 19-23, (In Chinese with English abstract).

87. Zhao, Y.; Wang, Q.F.; Sun, X.; Li, G.J. Characteristics of Ore-Forming Fluid in the Zhenyuan Gold Orefield, Yunnan Province, China. J. Earth Sci. 2013, 24, 203-211. [CrossRef]

88. Zhang, H.C.; Chai, P.; Zhang, H.R.; Hou, Z.Q.; Chen, S.M.; Sun, Y.B.; Peng, Q. Two-Stage Sulfide Mineral Assemblages in the Mineralized Ultramafic Rocks of the Laowangzhai Gold Deposit (Yunnan, SW China): Implications for Metallogenic Evolution. Resour. Geol. 2019, 69, 270-286. [CrossRef]

89. Yang, P.; Xu, Y.D.; Cheng, K.; Jiang, H.F.; Ma, Y.X. Metallogenic Conditions and Prospecting Orientation of Mojiang Jinchang Gold Deposit, Yunnan Province, China. Acta Mineral. Sin. 2013, 33, 585-591, (In Chinese with English abstract).

90. Li, Y.; Jin, S.C.; Yu, G.J. Istope geochemistry and genesis of Mojiang gold deposit, Yunnan. Geol. Geochem. 1998, 4, 15-20, (In Chinese with English abstract).

91. Zhang, Z.L.; Zhang, S.F.; Yuan, H.H.; He, D.L. An isotope geology and origin study of the Jinchang gold deposit Mojiang, Yunnan. J. Chengdu Coll. Geol. 1987, 4, 29-41, (In Chinese with English abstract).

92. Xie, G.Q.; Hu, R.Z.; Ni, P.; Su, W.C. Geochemical characteristics in gold-bearing quartz in the Mojiang gold deposit and their indications. Acta Mineral. Sin. 2001, 21, 613-618, (In Chinese with English abstract).

93. Xiong, Y.Q. The Ore-Forming Processes of Jinchang Hydrothermal Gold and Nickle Deposit, Mojiang, Western Yunnan, China. Master Thesis, China University of Geosciences, Beijing, China, 2014. (In Chinese with English abstract).

94. Zhang, H.T.; Yang, J.R.; Xue, D.Z. The hydrothermal type gold deposit in altered ultrabasic rock-A discussion about the origin of Jinchang gold deposit. Geol. Yunnan 1984, 4, 311-325, (In Chinese with English abstract).

95. Zhu, L.H.; Qi, X.X.; Peng, S.B.; Li, Z.Q. Evolution of ore forming fluid of Daping gold deposit in Ailaoshan tectonic zone, Southeast Tibet. Acta Petrol. Sin. 2011, 27, 3395-3408, (In Chinese with English abstract).

96. Shi, G.Y.; Sun, X.M.; Zhang, Y.; Xiong, D.X.; Zhai, W.; Pan, W.J.; Hu, B.M. H-O-C-S isotopic compositions of ore-forming fluids in Daping gold deposit in Ailaoshan gold belt, Yunnan Province, China. Acta Petrol. Sin. 2010, 26, 1751-1759, (In Chinese with English abstract).

97. Li, X.J. Metallogenic Mechanism and Prospecting Orientation of Daping Gold Deposit in Yuanyang, Yunnan Province. Master Thesis, China University of Geosciences, Beijing, China, 2017. (In Chinese with English abstract).

98. Han, R.S.; Jin, S.C.; Lei, L. Geochemistry of ore-forming hydrothermal system of Daping reworked gold deposit Yuanyang, Yunnan. Acta Mineral. Sin. 1997, 17, 337-344, (In Chinese with English abstract).

99. Li, D.M.; Li, B.H. The mineralization of the gold deposits in the Ailao Mountains, Yunnan. Sediment. Geol. Tethyan Geol. 2000, 1 , 60-78, (In Chinese with English abstract).

100. Ge, L.S.; Deng, J.; Li, H.G.; Yang, L.Q.; Zhang, W.Z.; Yuan, S.S.; Xing, J.B. Superposed mineralization in Daping Au-Cu-Ag-Pb deposit, Yunnan province: Evidences from geology, fluid inclusions and stable isotopes. Acta Petrol. Sin. 2007, 23, 2131-2143, (In Chinese with English abstract).

101. Yuan, S.S.; Ge, L.S.; Guo, X.D. Lead and sulfur isotope characteristics and geological implication of Daping gold deposit. J. Guilin Univ. Technol. 2011, 31, 11-18, (In Chinese with English abstract).

102. Yuan, S.S.; Ge, L.S.; Lu, Y.M.; Guo, X.D.; Wang, M.J.; Wang, Z.H.; Zou, Y.L. Relationship between crust-mantle reaction and gold mineralization in Ailaoshan metallogenicbelt: A case study of Daping gold deposit in Yuanyang. Miner. Depos. 2010, 29, 253-264, (In Chinese with English abstract).

103. Yang, Y.Z.; Long, Q.; Hu, H.T.; Hieu, P.T.; Nguyen, T.B.H.; Chen, F.K. Geochemical characteristics and origin of lamprophyre in the Laichau area, northwestern Vietnam. Acta Petrol. Sin. 2013, 29, 899-911, (In Chinese with English abstract).

104. Chang, X.Y.; Zhu, B.Q. The application of lead isotopes to geochemical exploration in the Daping gold deposit, Yunnan Province. Acta Geosci. Sin. 2005, 26, 146-150, (In Chinese with English abstract).

105. Zhang, G.G. Study on Metallogenisis and Ore Structural-Controlling of the Ailaoshan Gold Mineralization Belt. Ph.D. Thesis, China University of Geosciences, Beijing, China, 2017. (In Chinese with English abstract).

106. Ying, H.L.; Liu, H.L.; Yang, X.Z.; Li, Z.W. Geology and origin of Tongchang gold deposit in Yunnan province. Geol. Resour. 2006, 15, 265-271, (In Chinese with English abstract).

107. Chen, Y.; Liu, J.L.; Tran, M.D.; Li, Y.C.; Bing, M.M. Regional metallogenesis of the Chang'an gold ore deposit in western Yunnan: Evidences from fluid inclusions and stable isotopes. Acta Geol. Sin. 2010, 84, 1401-1414.

108. Li, S.H.; Zhang, J.; Deng, J.; Wang, H.; Liu, J.T.; Zhao, K. The characteristics of ore-forming fluid and genetic type of the Chang' an gold deposit in southern Ailaoshan metallogenic belt. Acta Petrol. Sin. 2011, 27, 3777-3786, (In Chinese with English abstract).

109. Li, W.; Li, Z.L.; Shi, G.Y. Characters of metamorphic fluid of Ailaoshan, Yunnan. Acta Petrol. Sin. 2000, 16, 649-654, (In Chinese with English abstract).

110. Zhang, C.; Liu, Y.; Liu, X.D.; Feng, J.Q.; Huang, T.; Zhang, Q.; Wang, X.D. Characteristics of sulfur isotope geochemistry of the Xincheng gold deposit, Northwest Jiaodong, China. Acta Petrol. Sin. 2014, 30, 2495-2506, (In Chinese with English abstract). 
111. Li, S.H.; Zhang, J.; Yang, L.Q.; Wang, H. Origin of metallogenetic materials of the Chang'an gold deposit in the southern Ailaoshan belt: Evidence from sulfur and lead isotopic composition. Geoscience 2013, 27, 879-887, (In Chinese with English abstract).

112. ECUT (East China University of Technology). Research Report of the Prospecting Direction and Metallogenic Model of the Chang'an Gold Deposit in Jinping County, Yunnan Province; ECUT: Jiangxi, China, 2007.

113. Bi, X.W.; Hu, R.Z.; He, M.Y. Characteristics of ore-forming fluid of three gold deposits in Ailaoshan gold metallogenic belt. Acta Mineral. Sin. 1997, 17, 435-441, (In Chinese with English abstract).

114. Xiong, D.X.; Sun, X.M.; Zhai, W.; Shi, G.Y.; Wang, S.W. $\mathrm{CO}_{2}$-Rich Fluid Inclusions in Auriferous Quartz Veins from the Daping Ductile Shear Zone Hosted Gold Deposit in Yunnan Province, China, and Its Implications for Gold Mineralization. Acta Geol. Sin. 2007, 5, 640-653, 721, (In Chinese with English abstract).

115. Du, D.Y. Ore-Forming Fluids Characteristics of the Daping Gold Deposit, Western Yunnan, China. Master Thesis, China University of Geosciences, Beijing, China, 2015. (In Chinese with English abstract).

116. Xin, W. Research on Cenozoic Au-Cu-Mo Metallogenesis in Ailaoshan-Honghe Metallogenic Belt, Yunnan Province. Ph.D. Thesis, Jilin University, Jilin, China, 2019. (In Chinese with English abstract).

117. Deng, J.; Wang, C.M.; Li, G.J. Style and process of the superimposed mineralization in the Sanjiang Tethys. Acta Petrol. Sin. 2012, 28, 1349-1361, (In Chinese with English abstract).

118. Taylor, H. Oxygen and hydrogen isotope relationships in hydrothermal mineral deposits. In Geochemistry of Hydrothermal Ore Deposits; John Wiley \& Sons Inc.: New York, NY, USA, 1997; pp. 229-302.

119. Taylor, B.E.; Eichelberger, J.C.; Westrich, H.R. Hydrogen isotopic evidence of rhyolitic magma degassing during shallow intrusion and eruption. Nature 1983, 306, 541-545. [CrossRef]

120. Taylor, H.P. The application of oxygen and hydrogen isotope studies to problems of hydrothermal alteration and ore deposition. Econ. Geol. 1974, 69, 843-883. [CrossRef]

121. Cao, H.W.; Li, G.M.; Zhang, R.Q.; Zhang, Y.H.; Zhang, L.K.; Dai, Z.W.; Zhang, Z.; Liang, W.; Dong, S.L.; Xia, X.B. Genesis of the Cuonadong tin polymetallic deposit in the Tethyan Himalaya: Evidence from geology, geochronology, fluid inclusions and multiple isotopes. Gondwana Res. 2021, 92, 72-101. [CrossRef]

122. Shimazaki, H.; Shimizu, M.; Nakano, T. Carbon and oxygen isotopes of calcites from Japanese skarn deposits. Geochem. J. 1986, 20, 297-310. [CrossRef]

123. Sheppard, S.M.F. Characterization and isotopic variations in natural waters. Rev. Miner. 1986, 16, $165-183$.

124. Rollinson, H.R. A terrane interpretation of the archean limpopo belt. Geol. Mag. 1993, 130, 755-765. [CrossRef]

125. Mao, J.W.; Wang, Z.L.; Li, H.M.; Wang, C.Y.; Chen, Y.C. Carbon and oxygen isotope components in the permian basalt-hosted copper deposit in Ludian area, Yunnan: Implication for mineralization process. Geol. Rev. 2003, 49, 610-615, (In Chinese with English abstract).

126. Liu, J.M.; Ye, J.; Xu, J.H.; Sun, J.G.; Shen, K. C-O and Sr-Nd isotopic geochemistry of carbonate minerals from gold deposit in East Shandong, China. Acta Petrol. Sin. 2003, 19, 775-784, (In Chinese with English abstract).

127. Zhang, J.; Yang, Y.; Hu, H.Z.; Wang, Z.G.; Li, G.P.; Li, Z.L. C-S-Pb isotope geochemistry of the Yindonggou orogenic type silver deposit in Henan Province. Acta Petrol. Sin. 2009, 25, 2833-2842, (In Chinese with English abstract).

128. Phillips, G.N.; Evans, K.A. Role of $\mathrm{CO}_{2}$ in the formation of gold deposits. Nature 2004, 429, 860-863. [CrossRef]

129. Zheng, Y.F. Theoretical modeling of stable isotope systems and its applications to geochemistry of hydrothermal ore deposits. Miner. Depos. 2001, 20, 57-70, (In Chinese with English abstract).

130. Brown, P.E.; Bowman, J.R.; Kelly, W.C. Petrologic and stable isotope constraints on the source and evolution of skarn-forming fluids at Pine Creek, California. Econ. Geol. 1985, 80, 72-95. [CrossRef]

131. Zheng, Y.F.; Hoefs, J. Carbon and oxygen isotopic covariations in hydrothermal calcites: Theoretical modeling on mixing processes and application to Pb-Zn deposits in the Harz Mountains, Germany. Miner. Depos. 1993, 28, 79-89. [CrossRef]

132. Zheng, Y.F.; Fu, B.; Zhang, X.H. Effects of magma degassing on the carbon and sulfur isotope compositions of igneous rocks. Chin. J. Geol. 1996, 31, 43-53, (In Chinese with English abstract).

133. Xiong, D.X.; Sun, X.M.; Zhai, W.; Shi, G.Y.; Wang, S.W. Graphite inclusions with high crystallinity in the gold-bearing quartz veins of the Daping gold deposit, Yunnan Province: Evidence for the involvement of metamorphic fluids of granulite facies in mineralization in the lower crust. Acta Geol. Sin. 2006, 80, 1448-1456, (In Chinese with English abstract).

134. Lu, Y.J.; Kerrich, R.; Kemp, A.I.S.; McCuaig, T.C.; Hou, Z.Q.; Hart, C.J.R.; Li, Z.X.; Cawood, P.A.; Bagas, L.; Yang, Z.M. Intracontinental Eocene-Oligocene porphyry $\mathrm{Cu}$ mineral systems of Yunnan, Western South China Block, China: Compositional characteristics, sources, and implications for continental collision metallogeny. Econ. Geol. 2013, 108, 1541-1576. [CrossRef]

135. Zindler, A.; Hart, S.R. Annual Review of Earth and Planetary Sciences. Chem. Geod. 1986, 14, 493-571.

136. Guo, Z.F.; Hertogen, J.; Liu, J.Q.; Pasteels, P.; Boven, A.; Punzalan, L.; He, H.Y.; Luo, X.J.; Zhang, W.H. Potassic magmatism in Western Sichuan and Yunnan Provinces, SE Tibet, China: Petrological and geochemical constraints on petrogenesis. J. Petrol. 2005, 46, 33-78. [CrossRef]

137. Hart, S.R. A large-scale isotope anomaly in the southern hemisphere mantle. Nature. 1984, 309, 753-757. [CrossRef]

138. Zhang, L.S.; Schärer, U. Age and origin of magmatism along the Cenozoic Red Rivershear belt, China. Contrib. Mineral. Petrol. 1999, 134, 67-85. [CrossRef]

139. Deng, W.M.; Huang, X.; Zhong, D.L. Petrological characteristics and genesis of Cenozoic alkali-rich porphyry in West Yunnan, China. Sci. Geol. Sin. 1998, 33, 412-425, (In Chinese with English abstract). 
140. Zhao, X.; Yu, X.H.; Mo, X.X.; Zhang, J.; Lu, B.X. Petrological and geochemical characteristics of Cenozoic alkali-rich porphyries and xenoliths hosted in western Yunnanprovince. Geoscience 2004, 18, 217-228, (In Chinese with English abstract).

141. Ohmoto, H. Systematics of Sulfur and Carbon Isotopes in Hydrothermal Ore Deposits. Econ. Geol. 1972, 67, 551-578. [CrossRef]

142. Ohmoto, H.; Rye, R.O. Isotope of sulfur and carbon. In Geochemistry of Hydrothermal Ore Deposits; John Wiley \& Sons Inc.: New York, NY, USA, 1979; pp. 509-567.

143. Butler, I.B.; Böttcher, M.E.; Rickard, D.; Oldroyd, A. Sulfur isotope partitioning during experimental formation of pyrite via the polysulfide and hydrogen sulfide pathways: Implications for the interpretation of sedimentary and hydrothermal pyrite isotope records. Earth Planet Sci. Lett. 2004, 228, 495-509. [CrossRef]

144. Liu, J.T.; Yang, L.Q.; Lü, L. Pulang reduced porphyry copper deposit in the Zhongdian area, Northwest China:Constrains by the mineral assemblages and the ore-forming fluid compositions. Acta Petrol. Sin. 2013, 29, 3914-3924, (In Chinese with English abstract).

145. Claypool., G.E.; Holser, W.T.; Kaplan, I.R.; Sakai, H.; Zak, I. The age curves of Sulfur and Ocygen isotopes in Marine Sulfate and their Mutual Inter pretation. Chem. Geol. 1980, 28, 199-260. [CrossRef]

146. Powell, R.; Will, T.M.; Phillips, G.N. Metamorphism in Archean greenstone belts-calculated fluid compositions and implications for gold mineralization. J. Metamorph. Geol. 1991, 9, 141-150. [CrossRef]

147. Haeussler, P.J.; Bradley, D.; Goldfarb, R.; Snee, L.; Taylor, C. Link between ridge subduction and gold mineralization in southern Alaska. Geology 1995, 23, 995-998. [CrossRef]

148. Rock, N.M.S.; Groves, D.I. Can lamprophyres resolve the genetic controversy over mesothermal gold deposits? Geology 1988, 16, 538-541. [CrossRef]

149. Deng, J.; Wang, Q.F.; Li, G.J.; Hou, Z.Q.; Jiang, C.Z.; Danyushevsky, L. Geology and genesis of the giant Beiya porphyry-skarn gold deposit, northwestern Yangtze block, China. Ore Geol. Rev. 2015, 70, 457-485. [CrossRef]

150. Hou, Z.Q.; Zhou, Y.; Wang, R.; Zheng, Y.C.; He, W.Y.; Zhao, M.; Evans, N.J.; Weinberg, R.F. Recycling of metal-fertilized lower continental crust: Origin of non-arc Au-rich porphyry deposits at cratonic edges. Geology 2017, 45, 563-566. [CrossRef]

151. Muntean, G.L.; Cline, J.S.; Simon, A.C.; Longo, A.A. Magmatic-hydrothermal origin of Nevada's Carlin-type gold deposits. Nat. Geosci. 2011, 4, 122-127. [CrossRef]

152. Hronsky, J.M.A.; Groves, D.I.; Loucks, R.R.; Begg, G.C. A unified model for gold mineralisation in accretionary orogens and implications for regional-scale exploration targeting methods. Miner. Depos. 2012, 47, 339-358. [CrossRef]

153. Griffin, W.L.; Begg, G.C.; O’Reilly, S.Y. Continental-root control on the genesis of magmatic ore deposits. Nat. Geosci. 2013, 6, 905-910. [CrossRef]

154. Rudnick, R.L.; Gao, S. Composition of the Continental Crust. In Treatise on Geochemistry; Pergamon: Oxford, UK, $2003 ;$ pp. 1-64.

155. He, W.Y.; Mo, X.X.; Yang, L.Q.; Xing, Y.L.; Dong, G.C.; Yang, Z.; Gao, X.; Bao, X.S. Origin of the Eocene porphyries and mafic microgranular enclaves from the Beiya porphyry Au polymetallic deposit, western Yunnan, China: Implications for magma mixing/mingling and mineralization. Gondwana Res. 2016, 40, 230-248. [CrossRef]

156. Cai, Y.F.; Wang, Y.J.; Cawood, P.A.; Fan, W.M.; Liu, H.C.; Xing, X.W.; Zhang, Y.Z. Neoproterozoic subduction along the Ailaoshan zone, South China: Geochronological and geochemical evidence from amphibolite. Precambrian Res. 2014, 245, 13-28. [CrossRef]

157. Tassara, S.; González-Jiménez, J.M.; Reich, M.; Schilling, M.E.; Morata, D.; Begg, G.; Saunders, E.; Griffin, W.L.; O’Reilly, S.Y.; Grégoire, M.; et al. Plume-subduction interaction forms large auriferous provinces. Nat. Commun. 2017, 8, 843. [CrossRef]

158. Lu, Y.J.; Mccuaig, T.C.; Li, Z.X.; Jourdan, F.; Hart, C.J.R.; Hou, Z.Q.; Tang, S.H. Paleogene post-collisional lamprophyres in western Yunnan, western Yangtze Craton: Mantle source and tectonic implications. Lithos 2015, 233, 139-161. [CrossRef]

159. Shi, G.Y.; Sun, X.M.; Pan, W.J.; Hu, B.M.; Qu, W.J.; Du, A.D.; Li, C. Re-Os dating of auriferous pyrite from the Zhenyuan super-large gold deposit in Ailaoshan gold belt, Yunnan Province, Southwestern China. Chin. Sci. Bull. 2012, 57, 4578-4586. [CrossRef]

160. Groves, D.I. The crustal continuum model for late-Archaean lode gold deposits of the YilgarnBlock, Western Australia. Miner. Depos. 1993, 28, 366-374. [CrossRef]

161. Groves, D.I.; Goldfarb, R.J.; Gebre-Mariam, M. Orogenic gold deposits: A proposedclassification in the context of their crustal distribution and relationship to other gold deposittypes. Ore Geol. Rev. 1998, 13, 7-27. [CrossRef] 